\title{
Modèles dosimétriques pour les radionucléides incorporés par les travailleurs*
}

\author{
C. CHEVALIER**, M. ROY***, J.-L. MALARBET****
}

RÉSUMÉ La dosimétrie des expositions internes aux radionucléides incorporés repose sur une connaissance aussi complète que possible du comportement des substances radioactives dans l'organisme. Ceci est essentiel pour interpréter les mesures d'activité faites dans le corps et ses émonctoires et les exprimer sous forme de quantités incorporées et de doses engagées. On décrit ici en termes généraux les voies d'incorporation, les transferts au sang, la distribution et la rétention dans les organes puis l'excrétion des radionucléides. L'ensemble de ces processus biocinétiques complexes est schématisé de façon à pouvoir être représenté par des modèles mathématiques accessibles au calcul. Depuis une dizaine d'années, la Commission internationale de protection radiologique a entrepris la révision systématique de toutes ses recommandations pour tenir compte de l'évolution des connaissances : révision des facteurs de pondération du risque stochastique, révision complète du modèle respiratoire, révision de nombreux facteurs d'absorption digestive, élaboration de modèles spécifiques de certains organes et de certains éléments. Cette démarche a conduit à réactualiser la plupart des coefficients de dose pour les travailleurs.

ABSTRACT Dosimetric models for intakes of radionuclides by workers.

Internal dosimetry from intakes of radionuclides is based, as accurately as possible, on the understanding of radioactive material behaviour in the body. This is most important to the interpretation of bioassay measurements for intake evaluation and committed dose assessment. General description is made of intake routes, blood uptake, distribution to organs, retention and excretion of radionuclides. Those complex biokinetic processes are modelled such as to allow mathematical calculations. Since about ten years, the International commission on radiological protection has been incorporating recent knowledge in this field and revising superseded recommendations : this is the case for stochastic risk weighting factors, respiratory tract models, digestive absorption factors, models for specific organs and specific nuclides. This work has produced a set of new dose coefficients for workers to replace most of the former ones.

\footnotetext{
* Le nombre de pages élevé de cet article est atypique du point de vue des critères d'acceptation de Radioprotection : ceci est da au fait que cet article était initialement prévu dans un numéro spécial consacré à la dosimétrie.

** EDF-GDF/Service Général de Médecine du Travail/Division MAN.

*** Institut de Protection et de Sûreté Nucléaire, CIPR Comité 2.

**** Institut de Protection et de Sureté Nucléaire, SDOS.
} 


\section{Introduction}

La CIPR, dans la publication 60 (ICRP, 1991) recommande, pour la protection radiologique, des limites de doses calculées sur un an, sur cinq ans ou sur la vie entière. Elle considère ici, d'abord, la dose efficace, parce que celle-ci est liée à la notion de détriment à l'organisme entier : risque stochastique de pathologie, réduction de la durée de vie, effets potentiels sur la descendance, etc. Ces effets sont susceptibles d'apparaître à des doses équivalentes faibles, pour chaque organe, bien inférieures à celles pour lesquelles on pourrait craindre des effets déterministes. C'est pourquoi, en dosimétrie interne, depuis l'abaissement de ces limites, la CIPR considère qu'il n'est plus nécessaire de se préoccuper de ces derniers.

Pour les travailleurs, la CIPR souhaite que la limite à appliquer ne soit pas celle de la vie entière, mais celle de valeurs discrètes de périodes de temps, n'excédant pas cinq ans. C'est la limite primaire de dose efficace de $20 \mathrm{mSv}$ qui est recommandée( ${ }^{(1)}$.

Pour assurer la surveillance de l'exposition interne, la CIPR fournit, pour la plupart des radionucléides, les doses efficaces délivrées à l'organisme par unité d'activité incorporée, DPUI. Celles ci sont calculées, pour chaque becquerel de radionucléide incorporé, en tenant compte de la période radioactive et de l'énergie libérée dans les tissus, à partir de modèles biocinétiques dont les paramètres s'appuient sur des observations humaines ou animales : la fraction que retient l'individu lorsqu'il inhale ou ingère un radionucléide, le cheminement de celui-ci du site de dépôt vers le sang puis du sang vers les organes, les vitesses de migration, puis enfin le taux d'élimination par les voies urinaires ou fécales.

Les voies d'incorporation, portes d'entrée des substances, sont surtout l'ingestion, dans le cas du public, et l'inhalation ou la blessure dans le cas des travailleurs. Cette dernière voie, bien que plus rare, peut donner lieu à des contaminations importantes.

Pour l'inhalation, un modèle récent pour les voies respiratoires de l'homme tient compte de nombreux facteurs, comme la granulométrie des substances inhalées et leur solubilité dans les milieux pulmonaires spécifique de la forme chimique à considérer.

Les biocinétiques de plusieurs radionucléides parmi les plus fréquemment rencontrés en radioprotection sont modélisées avec une précision accrue.

(1) Les limites primaires de la CIPR ont été adoptées dans les «Basic Safety Standards 》 de l'Agence internationale de l'énergie atomique puis dans la Directive européenne relative à la Radioprotection du 13 mai 1996. 
Après leur passage dans le sang, les actinides, les alcalino-terreux, l'iode, le fer et le plomb font l'objet de recyclages entre le sang et les organes fixateurs, squelette, foie, thyroïde, etc. Ceci conduit à la notion de période biologique «apparente» plus longue que celle de la rétention proprement dite dans chacun des organes.

\section{Principes généraux}

Les modèles biologiques utilisés dans le domaine de l'exposition interne ont pour but de fournir une description mathématique du cheminement de l'activité après l'incorporation d'un radionucléide. Pour traduire l'évolution dans le temps du comportement de cet élément chimique, il existe plusieurs méthodes, l'une d'entre elles est de considérer l'organisme comme composé de différents compartiments en série ou en parallèle. Chaque compartiment étant une entité biocinétique affectée d'un taux de transfert constant (cinétique de $1^{\text {er }}$ ordre) et pour tenir compte des changements de ces transferts au cours du temps, un même organe ou tissu peut alors être représenté par plusieurs compartiments.

Pour caractériser ces transferts, on définit :

- la période biologique, $T_{\mathrm{b}}$, laps de temps nécessaire pour que la quantité contenue dans le compartiment isolé diminue de moitié du fait du métabolisme (souvent exprimée en jours) ;

- la durée moyenne de résidence dans le compartiment (en jours),

$$
T_{\mathrm{m}}=T_{\mathrm{b}} / \operatorname{Ln} 2=T_{\mathrm{b}} / 0,693 ;
$$

- le taux de transfert, débit de sortie du compartiment (par jour),

$$
\lambda=\operatorname{Ln} 2 / T_{\mathrm{b}}=1 / T_{\mathrm{m}} \text {; }
$$

- la période effective $T_{\mathrm{e}}$ qui combine à la période biologique la période de décroissance radioactive de l'isotope considéré.

Ces paramètres permettent de calculer, à chaque instant, la rétention dans chaque compartiment ou chaque organe et l'irradiation subie. Cette rétention associée aux grandeurs physiques des rayonnements émis et à la masse de l'organe cible permet le calcul de la dose reçue.

La figure 1 représente le comportement général des substances pénétrant dans l'organisme et montre schématiquement les relations entre les modèles de dosimétrie interne :

- modèles des portes d'entrée pulmonaire ou digestive, blessure, qui définissent l'incorporation ;

- modèles systémiques qui décrivent, pour chaque espèce chimique, le devenir biocinétique de l'activité qui a pénétré dans les fluides de l'organisme (compartiment de transfert) à partir de la porte d'entrée. 


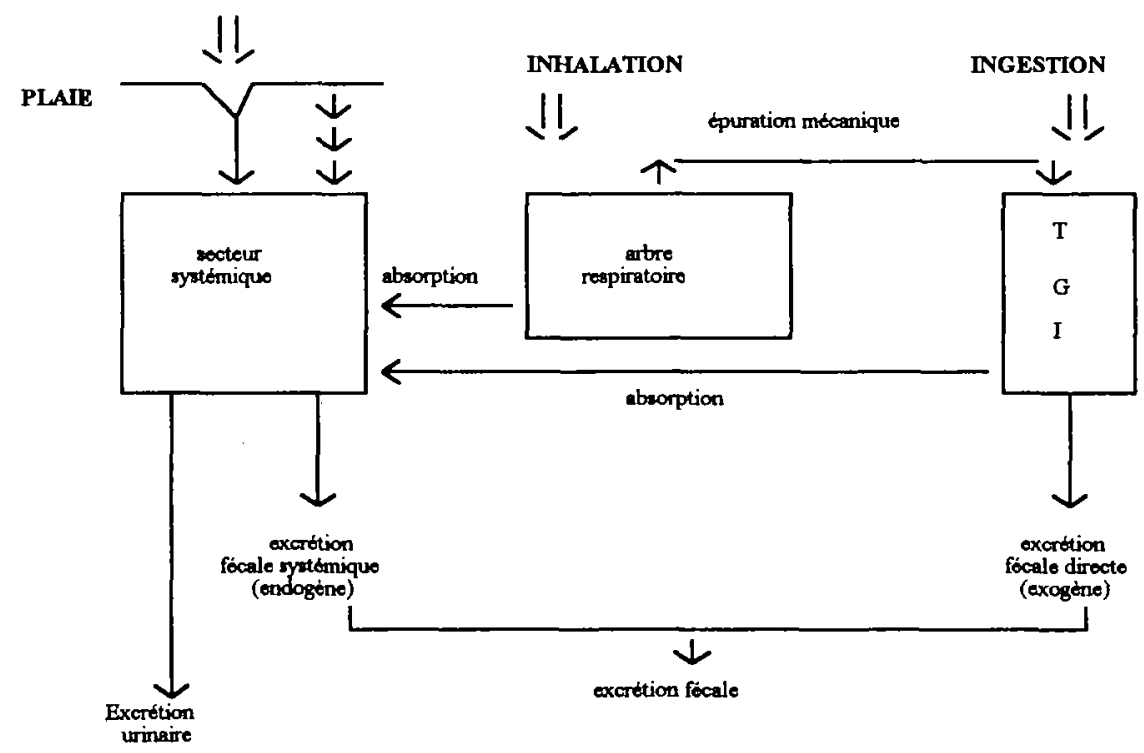

Fig. 1. - Diffêrents secteurs de l'organisme. Different parts of the body.

Ils permettent d'évaluer, pour un radio-isotope donné sous une forme chimique définie, les grandeurs suivantes.

\subsection{La dose absorbée}

L'énergie cédée par le rayonnement à l'unité de masse tissulaire est exprimée en gray (Gy, qui est égal à un joule par kilogramme) ; elle est délivrée ponctuellement. Comme il est rarement utile en radioprotection de calculer la dose en un point de l'organe, on préfère calculer la dose absorbée à l'organe qui est la dose moyenne absorbée dans un tissu ou dans un organe entier dont on connaît la masse. C'est une approximation introduite aux seules fins de la radioprotection.

\subsection{La dose équivalente}

Pour chaque tissu ou organe, la probabilité d'apparition d'un effet biologique dépend de la nature des rayonnements ou plus exactement de leur «nocivité ». Pour établir un système simple de protection, la CIPR introduit le concept de dose équivalente. Il permet de décrire un événement par une équivalence en photons de toutes énergies. C'est la notion de dose équivalente. Un facteur de pondération, $W_{\mathrm{R}}$, a été attribué à chaque rayonnement. Sa valeur est liée à la nature de l'émission et à son énergie. Ce facteur, établi pour la protection des 
effets stochastiques (faibles doses), est représentatif des valeurs de l'efficacité biologique relative (EBR) pour l'induction de cancers. La dose absorbée, pondérée par ce facteur $W_{\mathrm{R}}$, permet de calculer une valeur de dose produisant pour tous les types de rayonnements un effet de même importance. Elle est calculée pour chaque organe. Dans le cas de la contamination interne, on associe à la dose équivalente la notion de dose équivalente engagée pour tenir compte de la rétention du radionucléide dans l'organisme. L'engagement sera fait sur 50 ans pour les travailleurs et 70 ans pour les personnes du public.

\section{Le mode de calcul des doses engagées aux organes et à l'organisme entier est donné en annexe.}

\subsection{La dose efficace}

La connaissance des sensibilités relatives des divers organes aux cancers radioinduits, a été obtenue soit à partir d'études animales, soit par l'étude de populations irradiées telles que celles des survivants des bombardements d'Hiroshima et de Nagasaki. Les études récentes ont permis de mieux définir les cellules qui ont la plus grande probabilité d'être affectées par l'irradiation et d'attribuer à chaque tissu et organe un facteur de risque stochastique, représentatif du détriment global (effet cancer, effets génétiques et raccourcissement de la durée de vie). Ce facteur, appelé $W_{\mathrm{T}}$, permet pour chaque organe d'estimer sa contribution relative au risque stochastique total. Les valeurs attribuées à ces facteurs de pondération ont été révisées récemment dans la CIPR 60 (ICRP, 1991) ; elles figurent dans le tableau I.

La somme des doses équivalentes pondérées à l'aide des facteurs $W_{\mathrm{T}}$ est appelée dose efficace ou dose efficace engagée, $E$ (Sv) lorsqu'elle se rapporte à la contamination interne.

\subsection{Les rétentions et les excrétions}

Ces modèles permettent aussi de prévoir pour différentes dates après l'incorporation, les fractions qui seront retenues dans certains organes ou dans l'organisme entier, ainsi que les quantités qui seront excrétées par voie urinaire et fécale. Par comparaison avec les rétentions et les excrétions mesurées chez un individu, on pourra remonter à l'estimation de la quantité incorporée.

Au fur et à mesure que progresse la connaissance des données physiologiques, les modèles sont adaptés et deviennent plus aptes à satisfaire au mieux les objectifs précédents. Dans cette optique, il faut noter l'intérêt que peut présenter l'étude prolongée, dans le temps, de cas de contaminations vraies, et l'utilisation méthodique du retour d'expérience.

En devenant plus réalistes, les modèles deviennent aussi plus complexes, donc plus difficiles à traiter par le calcul. Par exemple, la CIPR, depuis sa publication 56 (ICRP, 1989) fait intervenir, de manière générale, le recyclage de l'activité entre plusieurs compartiments. 


\section{TABLEAU 1}

Facteurs de pondération $W_{T}$ de la publication 60 de la CIPR (ICRP, 1991).

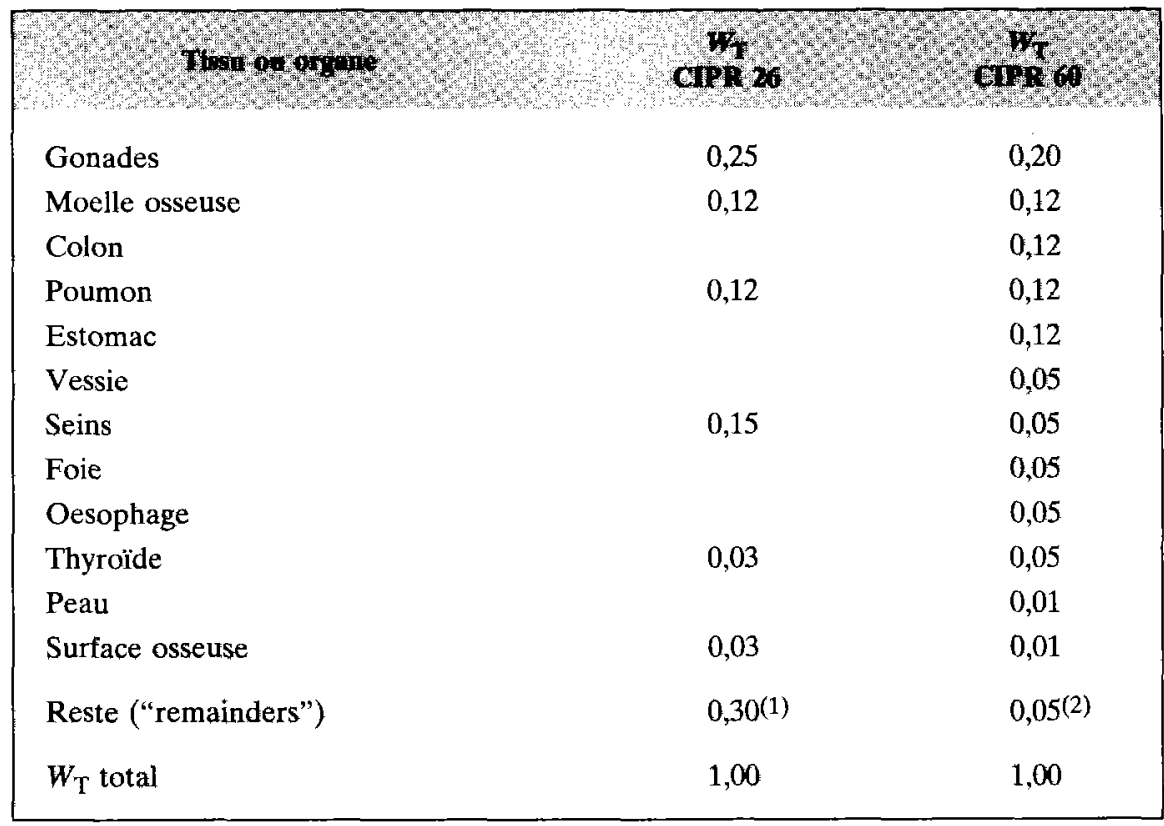

(1) Le « reste » est réparti uniformément entre chacun des 5 autres organes les plus exposés avec un $W_{\mathrm{T}}$ de $0,30 / 5=0,06$.

(2) Le « reste" constitué des organes suivants (cerveau, surrénales, intestin grêle, reins, muscle, pancréas, rate, thymus, utérus, région respiratoire extra-thoracique) ne représente que $5 \%$ du risque. Dans le cas où l'un de ces dix organes reçoit une dose équivalente supérieure à la plus élevée de celle des douze organes spécifiés plus haut, on lui attribue la moitié des $5 \%$.

Nous décrirons les différents types de modèles utilisés pour l'estimation des incorporations ou des doses qui résultent de l'exposition interne après les mises à jour récentes faites par le Comité 2 de la CIPR, qui est chargé d'élaborer des méthodes d'application opérationnelle des limites primaires.

\section{Modèles des portes d'entrée}

\subsection{Modèle gastro-intestinal}

Le modèle actuel est encore celui qui a été recommandé par la publication 30 (ICRP, 1979). 


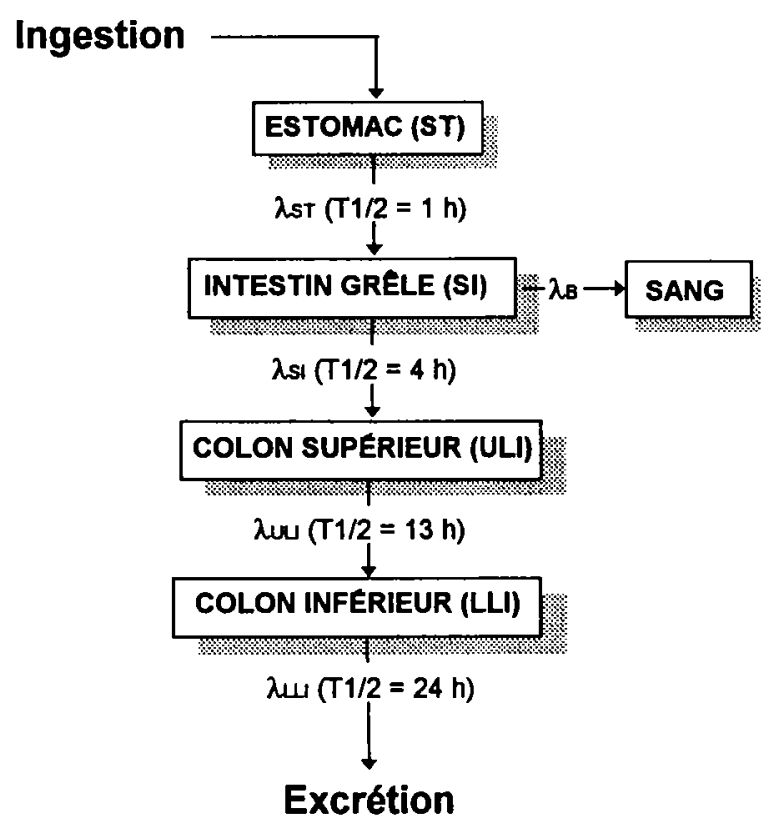

Fig. 2. - Modele gastro-intestinal.

Gastro-intestinal model.

Il comporte quatre compartiments représentés sur la figure 2 :

- l'estomac,

- l'intestin grêle,

- le colon pour sa partie supérieure,

- le colon pour sa partie inférieure.

Les durées moyennes de résidence dans les quatre compartiments sont respectivement de 1, 4,13 et 24 heures.

La séquence compartimentale et les durées moyennes de résidence sont valables pour tous les éléments sous toutes les formes chimiques. Au contraire, l'absorption des radionucléides vers le sang et les fluides extra-cellulaires (qui est supposée avoir lieu uniquement dans l'intestin grêle) et la valeur de la fraction $f_{1}$ d'activité qui est transférée au sang pour être absorbée dans le pool systémique, dépend du radionucléide considéré et de sa forme chimique(1).

(1) Le comité 2 de la CIPR a créé en mars 1996 un groupe de travail pour la révision du modèle digestif. Ses membres sont: H. Métivier, Président, J.D. Harrisson, J. Hendry, D. Nosske et J. Stubbs. 


$$
\begin{aligned}
& \text { C. CHEVALIER et al. } \\
& f_{1}=\lambda s / \lambda s+\lambda i g,
\end{aligned}
$$

avec: $\lambda s=$ constante de transfert vers le sang;

$\lambda i g=$ constante de transfert vers le colon.

Certaines valeurs de $f_{1}$ ont été revues récemment. Le tableau II regroupe les facteurs d'absorption digestive, $f_{1}$, dont les valeurs données dans la publication 68 (ICRP, 1994) diffèrent de celles de la publication 30 (ICRP, 1979) : les éléments concernés sont le thorium, les actinides, le cérium, le cobalt et le tellure.

Pour mieux suivre la réalité physiologique, des modifications plus profondes sont à envisager dans un proche avenir; on peut citer Simko et al. (1996) et Stubbs (1992).

\section{TABLEAU II}

Valeurs du facteur d'absorption digestive $f_{1}$ récemment modifiées pour passer des publications 30 et 61 de la CIPR (ICRP, 1979 et 1991)

\begin{tabular}{|c|c|c|c|c|c|c|}
\hline \multirow{2}{*}{ Clénents } & \multicolumn{3}{|c|}{ Publiations 30 et 61 de la CIPR } & \multicolumn{3}{|c|}{ Publication 68 de la CIPR } \\
\hline & Clusse & Inholation & Ingestion & Type & Inlalation & Ingestion \\
\hline \multirow[t]{3}{*}{$\mathrm{Pu}$} & W & $1 \times 10^{-3}$ & $1 \times 10^{-3}$ & M & $5 \times 10^{-4}$ & $1 \times 10^{-5}$ \\
\hline & $\mathrm{Y}$ & $1 \times 10^{-5}$ & $1 \times 10^{-4}$ & $\mathrm{~S}$ & $1 \times 10^{-5}$ & $1 \times 10^{-4}$ \\
\hline & & & $1 \times 10^{-5}$ & & & $5 \times 10^{-4}$ \\
\hline \multirow[t]{3}{*}{ Am } & W & $1 \times 10^{-3}$ & $1 \times 10^{-3}$ & M & $5 \times 10^{-4}$ & $1 \times 10^{-5}$ \\
\hline & & & & $S$ & $1 \times 10^{-5}$ & $1 \times 10^{-4}$ \\
\hline & & & & & & $5 \times 10^{-4}$ \\
\hline \multirow[t]{2}{*}{$\mathrm{Np}$} & W & $1 \times 10^{-3}$ & $1 \times 10^{-3}$ & M & $5 \times 10^{-4}$ & $1 \times 10^{-5}$ \\
\hline & & & & S & $1 \times 10^{-5}$ & $1 \times 10^{-4}$ \\
\hline \multirow{4}{*}{ Th } & & & & & & \\
\hline & W & $2 \times 10^{-4}$ & $2 \times 10^{-4}$ & M & $5 \times 10^{-4}$ & $1 \times 10^{-5}$ \\
\hline & $\mathrm{Y}$ & $2 \times 10^{-4}$ & & S & $1 \times 10^{-5}$ & $1 \times 10^{-4}$ \\
\hline & & & & & & $5 \times 10^{-4}$ \\
\hline \multirow[t]{2}{*}{$\mathrm{Ce}$} & W & $3 \times 10^{-4}$ & $3 \times 10^{-4}$ & $\mathbf{M}$ & $5 \times 10^{-4}$ & $5 \times 10^{-4}$ \\
\hline & Y & $3 \times 10^{-4}$ & & S & $5 \times 10^{-4}$ & \\
\hline \multirow[t]{2}{*}{$\mathrm{Co}$} & W & 0,05 & 0,05 & $\mathbf{M}$ & 0,05 & 0,1 \\
\hline & Y & 0,05 & 0,3 & $\mathrm{~S}$ & 0,05 & 0,05 \\
\hline \multirow[t]{2}{*}{$\mathrm{Te}$} & D & 0,2 & 0,2 & $\mathrm{~F}$ & 0,3 & 0,3 \\
\hline & W & 0,2 & & $\mathbf{M}$ & 0,3 & \\
\hline
\end{tabular}
à la publication 68 de la CIPR (ICRP, 1994). 
Ce sont :

- L'adjonction d'un compartiment pour la bouche et pour l'œsophage, et d'un compartiment à clairance plus lente dans le colon.

- L'absorption par le sang à partir de toutes les régions digestives et non plus seulement des régions supérieures.

- Des transferts plus complexes entre les compartiments.

- Des valeurs de $f_{1}$ différentes selon qu'un radionucléide est ingéré sous forme d'aliment liquide ou solide.

\subsection{Modèle pour les voies respiratoires}

Dans le modèle dosimétrique pour les voies respiratoires de la publication 30 (ICRP, 1979) les composés étaient rangés par défaut en trois classes de solubilité, D, W, Y (day, week, year). Le modèle décrit dans la publication 66 (ICRP, 1994) diffère du précédent sur plusieurs points :

- Le dépôt des aérosols est modélisé pour toutes les granulométries selon les modalités ventilatoires.

- La clairance se fait selon deux mécanismes distincts, le transport mécanique par les surfaces des voies aériennes, et l'absorption sanguine ou solubilisation qui est plus ou moins rapide.

- La principale nouveauté réside dans le fait que le poumon et les voies aériennes sont considérés comme des organes composites du point de vue du risque stochastique qui est donc inégalement réparti (il est à noter que l'on peut considérer comme très improbable de rencontrer des inhalations susceptibles de produire des effets somatiques précoces).

Les différentes étapes du modèle sont conçues soit pour utiliser des paramètres «par défaut » choisis comme les plus probables, à l'aide de données expérimentales, soit pour y substituer, chaque fois que ce sera possible, des valeurs réelles mesurées lors des observations faites aux postes de travail.

\subsubsection{Répartition régionale du risque stochastique}

En s'appuyant sur des études épidémiologiques et sur des études animales, on a observé que, lorsque tous les tissus pulmonaires reçoivent une même irradiation, ils ne présentent pas le même pourcentage de cancers radio-induits. Leur fréquence d'apparition permet d'en répartir le risque entre les différentes régions des voies respiratoires, et d'identifier les cellules «à risque » qui ont la plus grande probabilité d'être affectées par l'irradiation due aux radionucléides inhalés.

$\mathrm{Au}$ lieu de calculer la dose équivalente moyenne au poumon entier, on calcule donc les doses délivrées à chaque région respiratoire séparément. 
Les différents tissus de l'appareil respiratoire sont répartis en quatre ensembles anatomiques dont la connaissance détaillée permet le calcul dosimétrique pour chacun (Tableau III) :

- la région extra thoracique, subdivisée en ET1, nez antérieur non tapissé de muqueuse et ET2, nez postérieur, pharynx et larynx, c'est à dire voies oronasales ciliées, et leurs ganglions lymphatiques associés, $\mathrm{LN}_{\mathrm{ET}}$,

- les grosses bronches, BB, des générations 1 à 8 de Weibel,

- les bronchioles, bb, des générations de 9 à 16 ,

- les régions d'échanges gazeux, AI, alvéoles et interstitium pulmonaires, et les ganglions thoraciques, $\mathrm{LN}_{\mathrm{TH}}$.

Dans ces tissus, les cellules à risque sont surtout distribuées :

- dans la couche basale de l'épithélium stratifié extra thoracique (cellules basales),

- dans tout l'épithélium cilié bronchique (cellules basales et cellules sécrétoires),

- dans tout l'épithélium bronchiolaire (cellules sécrétoires de Clara),

- uniformément dans tout le tissu alvéolo-interstitiel (cellules endothéliales et pneumocytes II) et dans les ganglions.

L'évaluation des doses délivrées à chacun des tissus à risque dépend surtout de la quantité de radionucléide transportée avec l'air, de la fraction qui en

TABLEAU III

Pondération du détriment dans les voies respiratoires, de la publication 66 de la CIPR (ICRP, 1994)

\begin{tabular}{|c|c|c|}
\hline 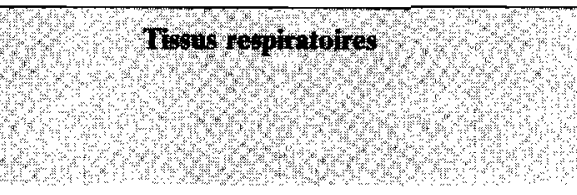 & $\begin{array}{l}\text { CIPA } 60 \\
\text { Wotnment total }\end{array}$ & 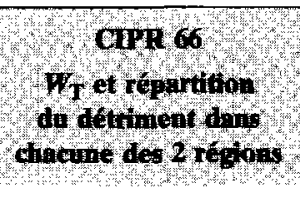 \\
\hline Extra-thoraciques (1/2 des « remainders ») & 0,025 & \\
\hline Nez antérieur (ET1) & & 0,001 \\
\hline Nez, bouche, pharynx, larynx (ET2) & & 0,998 \\
\hline Ganglions lymphatiques ( $\left.\mathrm{LN}_{\mathrm{ET}}\right)$ & & 0,001 \\
\hline Thoraciques (poumon) & $\mathbf{0 , 1 2}$ & \\
\hline Grosses bronches, G0-G8 (BB) & & 0,333 \\
\hline Bronchioles, G9-G16 (bb) & & 0,333 \\
\hline Alvéoles, interstitium (Al) & & 0,333 \\
\hline Ganglions lymphatiques $\left(\mathrm{LN}_{\mathrm{TH}}\right)$ & & 0,001 \\
\hline
\end{tabular}


est déposée à la surface des voies aériennes, et de la capacité des tissus à transporter ces substances ou à les retenir, les combiner chimiquement ou les solubiliser.

\subsubsection{Dépôt des radionucléides inhalés}

Le modèle de dépôt décrit les voies aériennes comme une série de filtres où se déposent les particules selon leur taille, durant l'inhalation et l'expiration (Fig. 3). L'efficacité de chaque région-filtre fait appel à des notions physiques : - les mécanismes thermodynamiques de diffusion auxquels sont soumises les particules de diamètre inférieur à environ $0,5 \mu \mathrm{m}$, pour lesquelles le diamètre thermodynamique est proche du diamètre géométrique.

- les mécanismes aérodynamiques de sédimentation et impaction auxquels obéissent les particules de diamètre supérieur à environ $0,5 \mu \mathrm{m}$, pour lesquelles le diamètre aérodynamique fait intervenir la densité particulaire.

On définit pour caractériser la granulométrie des aérosols les Diamètres Médians en Activité Thermodynamique, DTMA, et Aérodynamique, DAMA, pour lesquels $50 \%$ de l'activité de l'aérosol se trouve dans les particules de diamètre plus petit et $50 \%$ de l'activité dans les particules de diamètre plus grand.

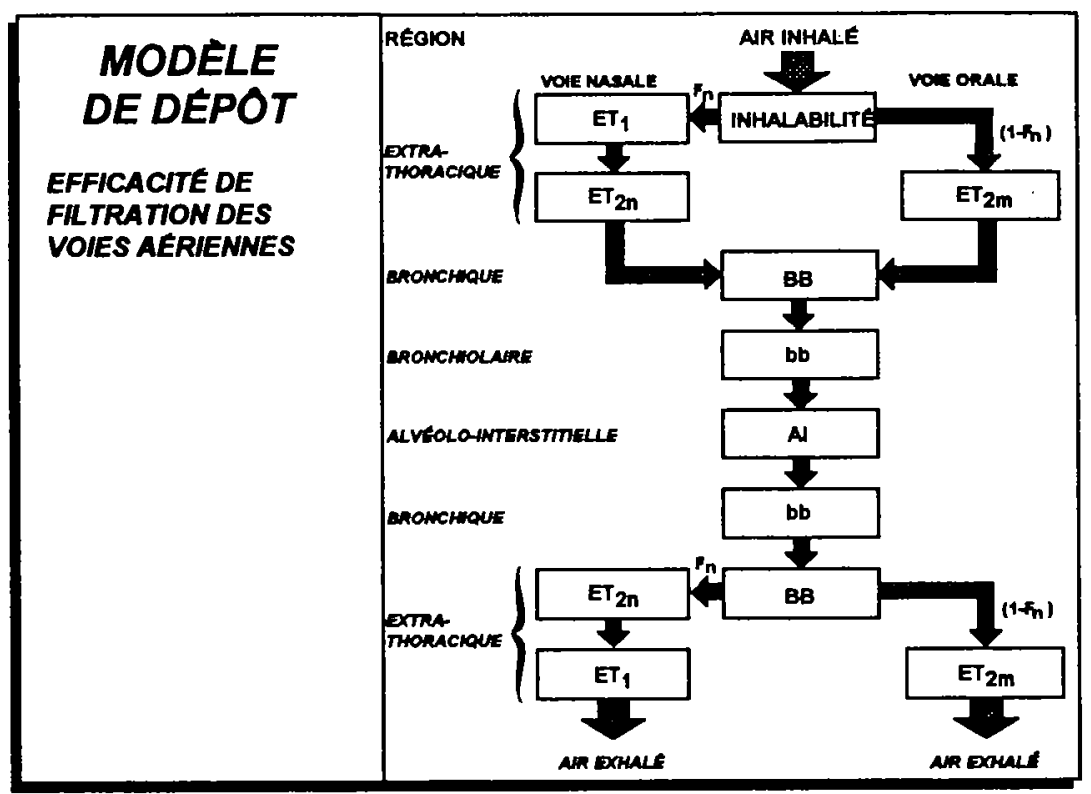

Fig. 3. - Modèle de dépôt des aérosols Aerosol deposition model 


\section{Aérosols}

Pour les aérosols, sont établies des équations et des courbes de dépôt standard, validées par des études expérimentales, et données chez l'homme pour toutes les tailles de particules allant de 0,0005 à $100 \mu \mathrm{m}$, dans chaque région respiratoire de l'individu de référence.

La fraction déposée dans les voies respiratoires d'un radionucléide inhalé varie donc selon sa granulométrie dans le cas d'un aérosol, et selon sa solubilité dans le cas d'un gaz; ceci explique, en partie, l'éventail des DPUI qui en résultent.

Pour les atmosphères de travail les valeurs de dépôt sont établies pour un aérosol dont le DAMA standard est de $5 \mu \mathrm{m}$, tandis que pour l'environnement il est de $1 \mu \mathrm{m}$.

\section{Gaz et vapeurs}

Un modèle particulier est développé pour les gaz et les vapeurs. La situation est différente de celle des aérosols qui était gouvernée par la distribution des tailles. Ici, c'est la chimie du composé qui intervient. On a déterminé 3 classes prenant en compte la solubilité et la réactivité avec les tissus :

- La classe SR-0 des composés insolubles et non réactifs, gaz inertes, pour lesquels il n'y a presque pas de transfert de l'activité inhalée. C'est l'irradiation externe qui comporte aussi l'irradiation intra pulmonaire, qui prime sur la contamination interne (gaz rares, azote).

- La classe SR-1 des composés solubles ou réactifs qui sont retenus dans les tissus respiratoires et transférés au sang. Sans données particulières, on admet la répartition suivante : $10 \%$ dans ET1, $20 \%$ dans ET2, $10 \%$ dans BB, $20 \%$ dans bb et $40 \%$ dans $\mathrm{AI}$. La quantité transférée au sang peut être inférieure ou égale à $100 \%$ de l'activité inhalée. Elle est de type «V $\mathrm{V}$ (very fast), équivalent à une injection intraveineuse pour un gaz très soluble, (iodure de méthyle gazeux, eau tritiée, monoxyde de carbone) et de type « $F$ » (fast), pour un gaz réactif avec ces tissus (oxygène, dioxyde de soufre, dioxyde d'azote, iode gazeux, disulfure de carbone, tétraoxyde de ruthénium).

- La classe SR-2 des composés très solubles ou très réactifs : le transfert au sang de l'activité inhalée intervient en totalité dans la région extra-thoracique et il est de type « V» instantané (tritium ozone, acide fluorhydrique, dioxyde de carbone, acétone).

\subsubsection{Clairance ou épuration des substances inhalées}

Dans chacune des régions respiratoires, la clairance varie avec le temps. Pour rester dans un système de cinétiques de premier ordre, ce mécanisme est représenté par un ensemble de plusieurs compartiments s'épurant à débits constants. 
Cette épuration résulte de la compétition entre deux mécanismes complémentaires :

- le transport par les cellules de surface et les macrophages vers les voies digestives, $d(t)$, et lymphatiques, $I(t)$. C'est la clairance mécanique.

- l'absorption par les tissus et le transfert vers le sang, $s(t)$. C'est la solubilisation.

La fraction épurée par unité de temps est donc la somme des deux :

$$
C(t)=d(t)+I(t)+s(t) .
$$

\section{La clairance mécanique}

Elle se fait pour toutes les substances au même débit dans une région donnée. Les valeurs en sont estimées à partir d'observations et d'études expérimentales, ce sont celles d'une substance idéale totalement insoluble (Fig. 4) :

- Dans les régions ciliées les dépôts ET2, BB1 et bb1 sont soumis à la clairance muco-ciliaire rapide avec des périodes biologiques moyennes de 10 minutes, 100 minutes et 8 heures respectivement.

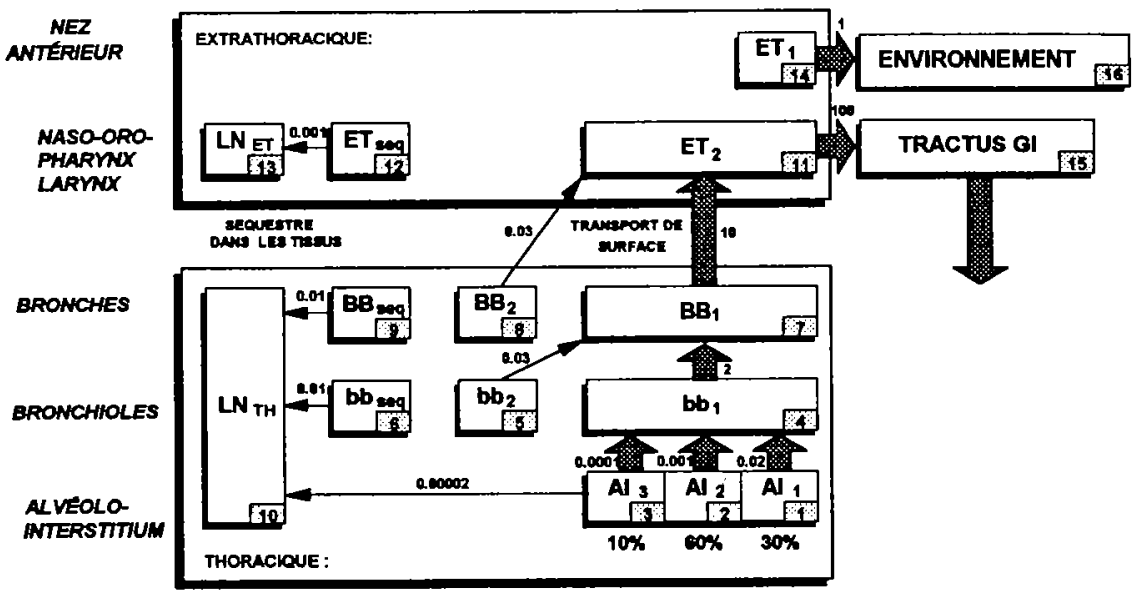

Fig. 4. - Modèle de clairance pulmonaire mécanique. Les taux de transfert à partir de chaque compartiment sont donnés par jour. Dans la région alvéolo-interstitielle, le dépôt est divisé en trois fractions déflnies par leurs vitesses de clairance.

Lung mechanical clearance model. Compartment transfer rates are expressed per day. In the alveolar interstitial region, deposit is divided into three fractions defined by their clearance rates. 
- Des fractions des dépôts bronchiques BB2 et bb2 peuvent être retenues à moyen terme après passage sous la couche épithéliale avec une période biologique de 23 jours.

- De très petites fractions sont «séquestrées » dans les parois des voies respiratoires. Ce sont les fractions ETseq, BBseq et bbseq, qui s'épurent lentement vers les ganglions lymphatiques.

Dans les régions alvéolaires, les périodes biologiques moyennes sont de 35 jours pour $\mathrm{Al}_{1}$, de 700 jours pour $\mathrm{Al}_{2}$ et de 7000 jours pour $\mathrm{Al}_{3}$. Les fractions du dépôt total dans $\mathrm{Al}$ sont respectivement de $30 \%, 60 \%$ et $10 \%$ pour $\mathrm{Al}_{1}, \mathrm{Al}_{2}$ et $\mathrm{Al}_{3}$.

Dans les ganglions lymphatiques thoraciques et extra-thoraciques qui n'ont pratiquement pas d'épuration mécanique mesurable, l'épuration se fait uniquement par la dissolution et le transport vers le sang.

\section{Le transport vers le sang après solubilisation}

La solubilisation se fait dans toutes les régions au même débit, mais le transport est spécifique de la substance déposée. Il peut être très rapide pour une substance à grande solubilité dans les milieux biologiques, mais suit dans le cas général au moins deux étapes: dissociation et solubilisation de la substance, puis passage dans le sang, sous une forme dissociée ou plus soluble.

Les paramètres de dissolution sont connus, pour nombre de substances, à partir d'observations et d'études expérimentales. Ils sont alors à utiliser directement dans les calculs. Cependant il est souvent commode d'utiliser des valeurs standards «par défaut » classés en 3 types, selon que le radionucléide appartient au type :

- F (fast) avec une période biologique d'absorption de 10 minutes,

- M (moderate) avec des périodes biologiques de $10 \mathrm{~min}(10 \%)$ et de 140 jours $(90 \%)$,

- S (slow) avec des périodes biologiques de $10 \mathrm{~min}(0,1 \%)$ et 7000 jours $(99,9 \%)$.

Il faut noter que dans les trois cas il existe une période de dissolution rapide, mais pour des fractions très différentes.

La détermination des incorporations et des doses délivrées à partir d'expositions à des radionucléides inhalés fait intervenir des paramètres divers tels que : la concentration dans l'air de l'aérosol ou du gaz radioactif, la durée pendant laquelle est respirée cette concentration. C'est pourquoi une place importante a été donnée aux paramètres physiologiques dépendant de l'âge. Les débits ventilatoires et les durées de résidence permettent de préciser l'exposition aux radionucléides inhalés. Les débits inhalés et leurs modalités par voie orale ou nasale déterminent les régions des voies aériennes où a lieu leur dépôt. Les masses de tissus-cibles des voies respiratoires permettent de calculer 
les doses absorbées. L'objectif est de fournir la dosimétrie non seulement des travailleurs mais aussi de tous les membres de la population. Des valeurs de référence pour tous ces paramètres sont donc définies pour les enfants âgés de 3 mois, 1 an, 5 ans, 10 ans, 15 ans, et pour les adultes. Une méthode est préconisée pour tenir compte de facteurs tels que le tabac, la pathologie respiratoire ou l'effet des polluants atmosphériques.

Un document technique sur la méthode permettant d'adapter le modèle pulmonaire en fonction de données spécifiques est en cours de rédaction par le Comité 2.

Un logiciel informatique a été développé afin de faciliter les calculs des doses par unité d'inhalation (Jarvis et Birchall, 1994). Il permet soit d'utiliser les paramètres standards, soit d'utiliser à la place des valeurs expérimentales telles que des granulométries d'aérosols mesurées sur un site, des paramètres de solubilité propres à un composé particulier, obtenus in vitro dans des liquides simulant le milieu pulmonaire ou in vivo chez l'animal et de faire une estimation plus réaliste et plus "spécifique " pour la surveillance de l'exposition des travailleurs dans des ateliers où l'empoussiérage est défini.

\subsection{Modèle de transfert à partir d'une blessure}

La CIPR n'a pas établi de modèle de transfert à partir d'une blessure. Le seul modèle actuellement connu est celui de Piechowski et al. (1992).

Les auteurs définissent trois compartiments de rétention au niveau de la plaie correspondant à trois fractions de l'activité initialement déposée $(Q)$ décrits dans la figure 5 :

$-Q_{1}$ : cette fraction, biologiquement inerte, n'est pas transférée, elle reste donc in situ;

$-Q_{2}$ : cette fraction est rapidement transférée par les brèches vasculaires $\left(T_{\mathrm{b}}=0,01 \mathrm{j}\right)$;

$-Q_{3}$ : cette fraction diffuse lentement dans les tissus mous environnants à partir desquels elle est transférée au sang, soit directement, soit après passage dans les ganglions lymphatiques.

Au plan de l'exposition systémique, seules les fractions $Q_{2}$ et $Q_{3}$ interviennent.

La fraction $Q_{2}$ est largement prédominante pour les composés solubles $\left({ }^{3} \mathrm{H}\right.$, $\mathrm{Cs}, \mathrm{I}$ ) et pour les composés insolubles (en particulier pour le plutonium). Outre les facteurs dosimétriques, les auteurs fournissent, en fonction du temps, des courbes de rétention systémique et/ou d'excrétion urinaire, permettant d'estimer l'activité de $Q_{2}$ à partir des mesures.

La fraction $Q_{3}$, dont le transfert est plus lent, n'est significative que pour les composés de solubilité intermédiaire. Son existence peut être suspectée si les 


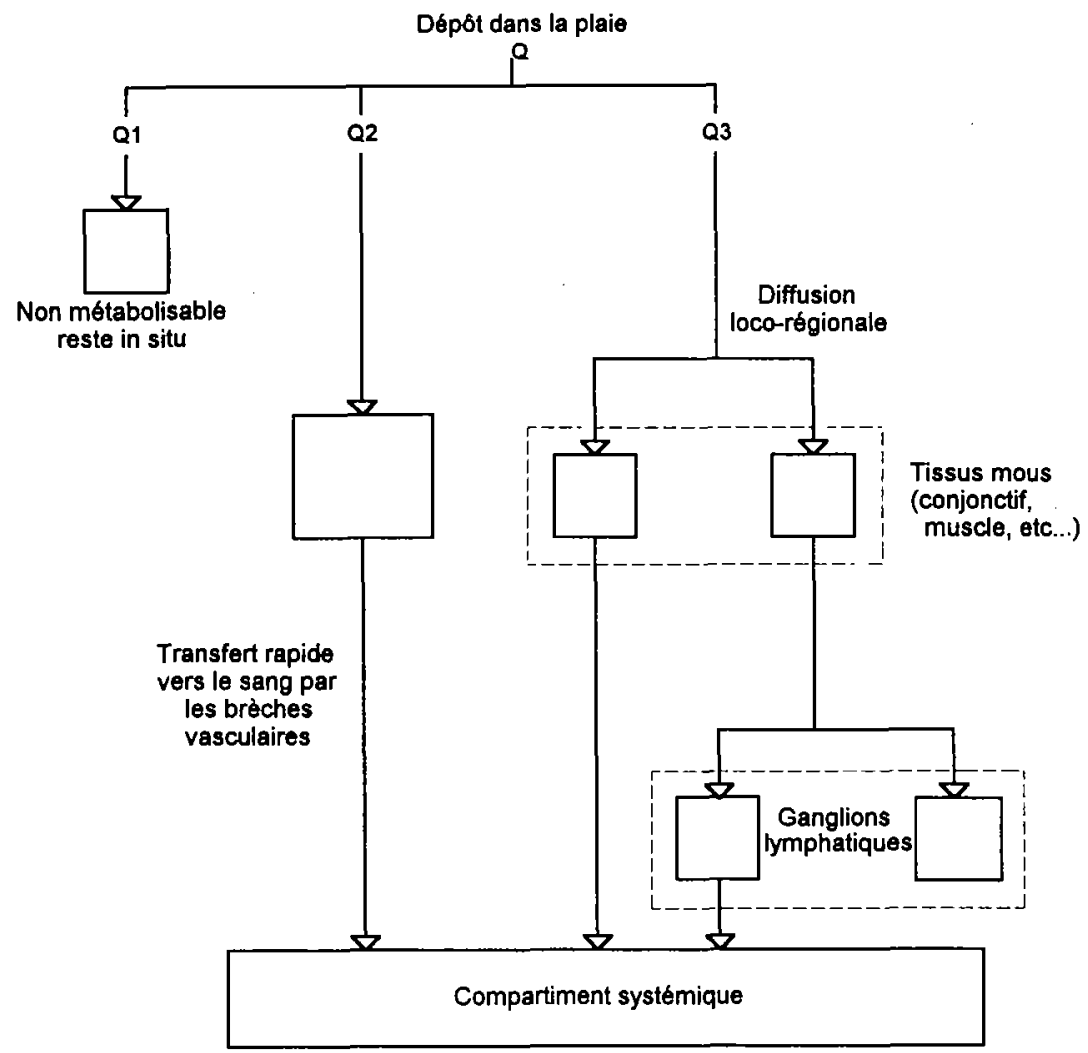

Fig. 5. - Schéma des transferts à partir d'une blessure contaminée.

Contaminated wound transfer model.

mesures d'excrétion urinaire s'écartent progressivement vers le haut des courbes théoriques correspondant à la fraction $Q_{2}$. On peut supposer qu'à terme, la totalité de l'activité de la fraction $Q_{3}$ sera transférée au sang, et utiliser l'hypothèse majorante que $Q_{3}$ est égale à l'activité mesurée au niveau de la plaie après excision.

\section{Modèles de biocinétiques systémiques}

Il est admis qu'il n'existe pas en général d'effet isotopique(1), donc tous les isotopes d'un même élément ont les mêmes biocinétiques. De plus, il est admis qu'un élément donné subit les mêmes biocinétiques quelle que soit la forme

(1) Un effet isotopique existe cependant pour le tritium. 
chimique sous laquelle il a été incorporé. Les éléments, chimiquement proches, sont regroupés en famille, alcalins, alcalino-terreux, actinides.

Les fluides de l'organisme (sang et lymphe) dans lesquels pénètre l'activité absorbée à partir des portes d'entrée, constituent le premier compartiment : c'est le compartiment de transfert, décrit dans la figure 6.

Ce compartiment, suivant le mode d'incorporation, est alimenté de manière plus ou moins prolongée à partir de la porte d'entrée. Son remplissage n'est jamais instantané sauf dans le cas d'une injection intraveineuse, d'une plaie (fraction $Q_{2}$ ) ou de l'inhalation d'un gaz très soluble. Il se vide avec une période biologique qui est, pour la plupart des éléments, égale à 6 heures soit 0,25 jour pour la publication 30 (ICRP, 1979).

Une partie de l'activité contenue dans le compartiment de transfert peut être directement excrétée de l'organisme tandis que le reste de l'activité se répartit dans les différents organes susceptibles de les fixer. Le processus physiologique réel inclue toujours un recyclage. Il est clair, par exemple, qu'un élément fixé dans un organe repasse par le sang avant d'être excrété par l'urine.

Comme on le verra plus loin avec plus de détails dans l'exemple des éléments ostéotropes, le plasma sanguin entretient des échanges plus ou moins rapides avec les tissus mous : peau, tissus sous-cutanés, muscle. Ils contribuent avec des vitesses variables à alimenter les autres organes. Une part de l'activité relarguée par ceux-ci est retournée au sang et recyclée par ce pool sérum-tissus mous, à nouveau vers ces mêmes organes.

Le processus est plus facile à réduire en équations sans tenir compte du recyclage et l'on a essayé souvent de le simplifier par un modèle non-recyclant, donnant des résultats approchés, utilisant la notion de période biologique apparente. Les périodes biologiques indiquées pour chaque compartiment

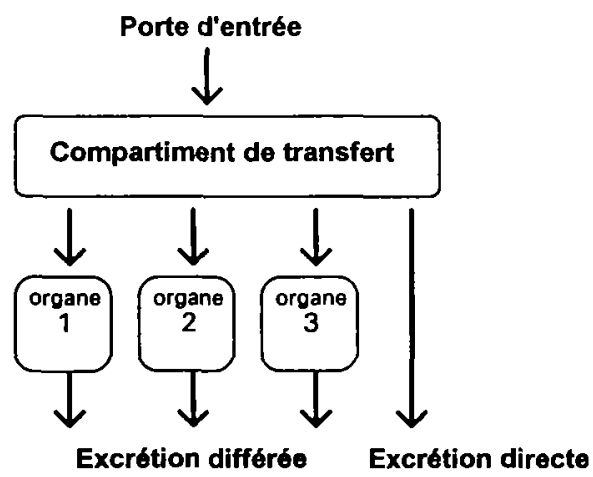

Fig. 6. - Rôle du compartiment de transfert.

Transfer compartment role. 
isolé, c'est-à-dire en l'absence de recyclage, diffèrent des périodes biologiques apparentes qui peuvent être estimées à partir de la rétention de l'activité dans chaque organe ou dans l'organisme entier. Ces dernières sont plus longues, et résultent le plus souvent d'une somme de fonctions exponentielles. En pratique, il est parfois possible d'utiliser cette méthode pour l'estimation de la dose, mais elle peut conduire à des erreurs importantes dans celle de la rétention et de l'excrétion à un temps précis après l'incorporation. Il faut alors s'astreindre à inclure les valeurs biocinétiques réalistes plutôt que la valeur brute de rétention dans les organes.

Les fonctions de rétention reposent largement sur l'expérimentation animale (étude de la variation de l'activité dans l'organisme après une injection intraveineuse, données d'autopsie pour l'étude de la répartition de l'activité dans les différents organes). Les données humaines obtenues par l'étude de cas de contaminations accidentelles ont été prises en compte chaque fois qu'elles étaient disponibles. Le foie et les voies d'excrétion ont été individualisés dans les modèles les plus récents pour calculer les doses aux organes pour lesquels un facteur de pondération a été défini dans la publication 60 . Ceci permet aussi une meilleure quantification des excrétions.

\subsection{Fonction de rétention systémique}

Dans la publication 30 (ICRP, 1979) la rétention systémique totale est explicitée sous la forme d'une fonction de rétention constituée d'une somme d'exponentielles :

$$
R(t)=\sum_{i=1}^{n} a_{i} \cdot \mathrm{e}^{-0,693 t / T_{i}}
$$

où $T_{i}$ sont les périodes biologiques exprimées en jours, $\sum_{i=1}^{n} a i=1$.

Cette fonction représente l'évolution, en fonction du temps, de l'activité contenue dans l'organisme entier après pénétration, à $t=0$, d'une activité unitaire dans le compartiment de transfert (équivalent à une injection intraveineuse). Valable pour tous les isotopes d'un même élément, elle ne tient pas compte de la décroissance radioactive.

Les différentes exponentielles ne correspondent qu'exceptionnellement à des organes définis, aussi l'équation de rétention doit-elle être explicitée. Un exemple simple est le Césium. Son équation de rétention $\left(0,10 \mathrm{e}^{-0,693 t / 2}+0,90 \mathrm{e}^{-0,693 t / 110}\right)$ comprend deux termes. Le Césium est réparti uniformément dans l'organisme mais, dans chaque organe ou tissu, $10 \%$ de l'activité sont retenus avec une période de 2 jours, tandis que les $90 \%$ restants sont retenus avec une période de 110 jours. Il n'y a pas d'excrétion directe à partir du compartiment de transfert.

Si l'on s'intéresse à un organe précis, il est nécessaire de dissocier, dans la fonction de rétention, la part qui revient à cet organe. 
La fonction de rétention du cobalt est :

$$
R(t)=0,5 \mathrm{e}^{-0,693 t / 0,5}+0,3 \mathrm{e}^{-0,693 t / 6}+0,1 \mathrm{e}^{-0,693 t / 60}+0,1 \mathrm{e}^{-0,693 t / 800} .
$$

Il est précisé en outre que la moitié du cobalt qui pénètre dans le compartiment de transfert est directement excrétée (première composante de la fonction) ; pour l'autre moitié, $5 \%$ sont fixés dans le foie et $45 \%$ dans le reste des organes ou tissus dans lesquels il se distribue uniformément. Pour une activité unitaire pénétrant dans le compartiment de transfert, la fonction de rétention dans le foie est donc:

$$
R(t) \text { foie }=0,03 \mathrm{e}^{-0,693 t / 6}+0,01 \mathrm{e}^{-0,693 t / 60}+0,01 \mathrm{e}^{-0,693 t / 800}
$$

(avec $0,03+0,01+0,01=0,05$ soit $5 \%$ ).

La fonction de rétention dans tout autre organe peut également être établie en tenant compte de sa masse relative par rapport à $68,2 \mathrm{~kg}$ (masse de l'organisme entier moins celle du foie).

Le calcul de la rétention dans l'organisme entier (ou dans un organe) à différentes dates après une injection intraveineuse, est simple, puisqu'il repose sur l'utilisation de la fonction de rétention systémique.

Ce calcul est moins simple après une incorporation par inhalation, par exemple, car l'activité ne pénètre que progressivement dans le compartiment de transfert à partir des différents compartiments de la porte d'entrée. L'expression mathématique de la rétention résultante, au temps $t$ après l'incorporation, est ainsi beaucoup plus complexe. Il convient, de plus, à chaque temps $t$, de rajouter l'activité résiduelle retenue au niveau de la porte d'entrée si l'on s'intéresse à l'activité totale contenue dans l'organisme.

La rétention s'exprime en $\mathrm{Bq}$ par $\mathrm{Bq}$ incorporé.

\subsection{Fonctions d'excrétion totale, urinaire et fécale}

\subsubsection{Fonction d'excrétion totale systémique}

Dans la publication 54 de la CIPR (1988) par souci de simplification, il est admis que l'activité éliminée à partir d'un organe (ou plus généralement d'un compartiment) est directement excrétée sans passage par le sang ni recyclage dans un autre organe.

Mathématiquement, la fonction exprimant le débit d'excrétion totale systémique est égale à la dérivée de la fonction de rétention systémique. Son expression est donc :

$$
E(t)=\sum_{i=1}^{n} \frac{0,693}{T_{i}} a_{i} \cdot \mathrm{e}^{-0,693 t / T_{i}}
$$


Comme la fonction de rétention systémique, la fonction d'excrétion résultante après une incorporation par inhalation ou ingestion est elle aussi mathématiquement plus complexe.

En définitive, l'excrétion journalière du jour $t$ après l'incorporation est égale à l'intégrale, entre $t-1$ et $t$, de la fonction d'excrétion résultante. L'excrétion totale journalière s'exprime en Bq par Bq incorporé.

\subsubsection{Fonctions d'excrétion urinaire et fécale}

Elles se déduisent simplement de la fonction d'excrétion totale systémique car il est admis que l'excrétion ne se fait que par les urines et les selles (la sueur et la salive ne sont pas prises en compte) et que le rapport de ces deux types d'excrétion est constant dans le temps. Pour chaque élément, la CIPR définit un facteur fu qui est la fraction de l'excrétion totale éliminée dans les urines. La fraction éliminée dans les selles est alors 1 - fu.

Lorsqu'elles existent, la CIPR conseille d'utiliser des fonctions d'excrétion fécale et urinaire, plus réalistes, obtenues expérimentalement chez l'homme. C'est le cas en particulier pour le plutonium. Le rapport de l'excrétion urinaire et fécale systémique n'est alors plus constant dans le temps.

Il est important de noter que l'excrétion fécale totale, après une incorporation par ingestion ou inhalation, est constituée non seulement de l'excrétion fécale systémique mais également de l'excrétion fécale directe de l'activité ingérée ou épurée mécaniquement de l'appareil respiratoire (fig. 1). Cette excrétion fécale directe est largement prédominante pendant plusieurs mois après une inhalation de composés peu ou moyennement transférables.

\subsection{Exemples de métabolismes systémiques}

\subsubsection{Le tritium et ses composés}

Le tritium dans l'environnement existe sous plusieurs formes : eau tritiée, tritium gazeux qui se transforme en eau tritiée par oxydation et molécules organiques tritiées.

L'eau tritiée: La caractéristique de l'eau tritiée est d'être bien absorbée par la peau intacte. En effet, dans une atmosphère contenant de la vapeur d'eau tritiée, l'absorption se fait pour $1 / 3$ au travers de la peau et pour $2 / 3$ par inhalation.

Quel que soit le mode d'incorporation, l'eau tritiée est totalement absorbée dans le sang $\left(f_{1}=1\right)$. Elle se répartit rapidement et uniformément dans l'eau libre corporelle sauf pour une faible fraction du tritium qui se lie aux molécules organiques (environ $3 \%$ ). Cette fraction est généralement négligée car elle ne contribue que pour environ $10 \%$ de la dose totale. 
La concentration du tritium dans tous les fluides de l'organisme (sang, urines) est la même. Elle décroît en fonction du temps après la fin de l'incorporation. On admet que le renouvellement de l'eau libre corporelle (environ $50 \mathrm{~kg}$ ) s'effectue avec une période biologique de 10 jours chez l'adulte.

Les molécules organiques tritiées: Le tritium fixé dans les molécules organiques alimentaires $\left(\mathrm{T}_{\text {orga }}\right)$, protéines, sucres, lipides etc. est aussi absorbé en totalité. La digestion de ces molécules les réduit à l'état de molécules plus petites qui seront absorbées. On admet que l'absorption digestive du tritium est totale $\left(f_{1}=1\right)$ et que $50 \%$ du tritium ainsi absorbé est échangé avec des atomes d'hydrogène de l'eau libre tandis que les $50 \%$ restants suivent le taux de renouvellement du carbone (liaison : $\mathrm{C}-\mathrm{H}$ ) dont la période biologique est de 40 jours chez l'adulte, publication 56 (ICRP, 1989). Il est possible que cette période soit sous-estimée dans des structures telles que le collagène, ou l'os. Les molécules marquées comme la thymidine tritiée pourraient aussi avoir une période très longue.

\subsubsection{L'iode}

L'iode est l'exemple type d'un élément se concentrant presque exclusivement dans un seul organe : la thyroïde.

L'iode après une incorporation est entièrement absorbé que ce soit par ingestion $\left(f_{1}=1\right)$ ou par dépôt dans les voies aériennes après une inhalation (type F). Une solution iodée déposée sur la peau est également bien absorbée mais cette absorption n'a pas été quantifiée.

Une partie de l'iode qui pénètre dans le sang se fixe au niveau de la thyroïde où il est incorporé dans les hormones thyroïdiennes en cours de synthèse. Le reste est éliminé par voie urinaire (Fig. 7).

Déjà, l'évidence de son recyclage est telle que le modèle de l'iode est le premier et le seul à l'inclure dans la publication 54 (ICRP, 1988). La fixation thyroïdienne, variable suivant les individus, est prise en moyenne égale à $30 \%$ de la charge sanguine. Elle ne dépend pas du sexe ni de l'âge (après 3 mois) mais de l'apport alimentaire en iode stable. Si cet apport est inférieur à 100 $\mu \mathrm{g} \mathrm{j}^{-1}$, la fixation est plus importante.

Chez l'adulte, l'iode intégré dans les hormones thyroïdiennes est éliminé de la thyroïde avec une période biologique de 80 jours (période apparente de 120 jours en raison du recyclage).

L'iode hormonal, libéré de la thyrö̈de, se distribue dans tout l'organisme dans lequel il est retenu, avec une période de 12 jours chez l'adulte. Vingt pourcent sont éliminés sous forme d'iode hormonal dans les selles tandis que les $80 \%$ restants retournent dans la circulation sous forme d'iode minéral après catabolisme des hormones thyroïdiennes. Cet iode est donc recyclé et 
subit le même métabolisme que l'iode initialement absorbé. Quel que soit l'âge, l'iode extra-thyroïdien est égal à $1 / 10$ de l'iode intra-thyroïdien.

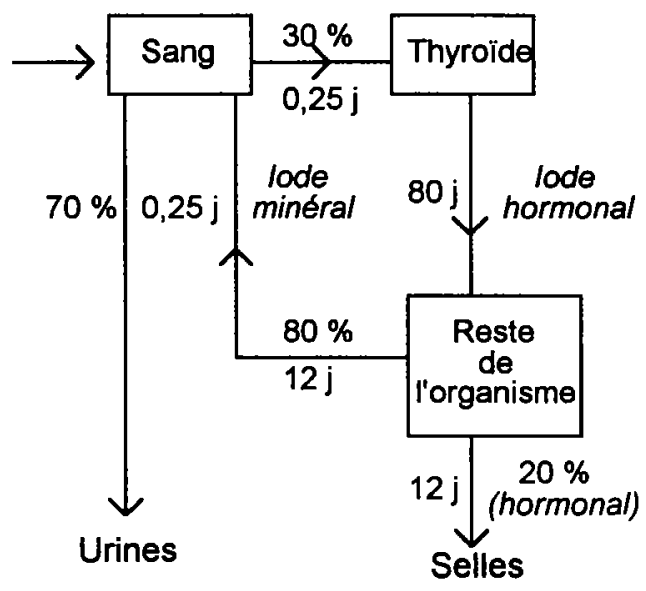

Fig. 7. - Modèle biocinétique de l'iode chez l'adulte. Iodine biokinetic model in adults.

\subsubsection{Le fer}

Le facteur d'absorption digestive $f_{1}$ est égal à 0,1 ; par inhalation, la plupart des composés, oxydes, hydroxydes et chlorures de valence 2 et 3 , sont moyennement solubles (type $M$ ).

La publication 53 (ICRP, 1987) décrit un modèle de rétention pour le fer qui est basé sur celui du MIRD Dose Report n 11 (Robertson et al., 1983). Ce modèle a été revu pour la publication 69 (ICRP, 1995) avec des données expérimentales plus complètes.

Après passage vers le sang, 1/3 du fer s'échange avec un compartiment rapide des tissus mous ( $18 \mathrm{~h}$ de période biologique) mais les $2 / 3 \mathrm{du}$ fer, liés à la transferrine, sont transportés vers la moelle osseuse hématopoiétique, avec une période de 160 minutes, et rendus au sang dans les hématies, dont le fer est contenu dans l'hémoglobine. Après destruction des hématies, dont la durée de vie est d'environ 120 jours, le fer retourne au plasma.

Environ $1 \mathrm{~g}$ de fer est stocké chez l'adulte dans la rate, la moelle osseuse, le foie et les tissus mous à échanges lents (système réticulo-endothélial et myoglobine des muscles). Dans le foie, un deuxième compartiment de transit vers l'intestin et le plasma est décrit. 
En dehors des pertes sanguines et des cellules d'autres tissus, l'excrétion du fer après recyclage est faible, et se fait surtout par les fèces $\left(\lambda=4\right.$ à $\left.5 \times 10^{4} \mathrm{j}^{-1}\right)$.

\subsubsection{Les éléments ostéotropes}

Certains radionucléides ont, comme le calcium, des affinités avec le tissu osseux auquel ils se lient pour des périodes très longues. Un modèle déjà complexe était proposé dans la CIPR 30 (ICRP, 1979) pour ces ostéotropes, émetteurs $\beta$, tels le strontium, ou émetteurs $\alpha$, tels le radium. Ce modèle montrait que, dans l'os, l'activité qui avait pénétré dans le sang pouvait se déposer sur les surfaces osseuses, comme dans le cas du thorium, ou à l'intérieur du volume osseux, comme dans le cas de l'uranium. En règle générale, les isotopes à vie courte (moins de 15 jours) restaient localisés à la surface, tandis que les isotopes à vie plus longue avaient la possibilité d'atteindre le volume osseux.

En 1989, la CIPR, dans la publication 56, a initié un nouveau modèle pour les ostéotropes basé sur de nombreuses données expérimentales. C'est le premier modèle qui permette une bonne précision aussi bien pour la dosimétrie que pour les calculs d'excrétions.

Dans le sang, l'activité est constamment recyclée avec les tissus fixateurs. Dans la circulation, le plasma est traité comme un pool uniforme d'échanges qui se font à des vitesses variables selon les radionucléides. Une partie importante entretient avec un compartiment de tissus mous ST0, des échanges rapides dont la période est variable suivant l'élément (quelques minutes à quelques heures). Ces tissus mous, muscles, peau, tissus sous-cutanés, à l'exception du foie et des reins, comportent aussi deux autres compartiments où de faibles fractions sont retenues: ST1, à moyen terme et ST2, à très long terme (quelques jours à quelques années).

Le foie est représenté par un ou deux compartiments, selon l'élément, l'un rapide, avec un cycle entéro-hépatique, l'autre plus lent, en relation avec le système réticulo-endothélial.

Le rein est représenté par deux compartiments, l'un d'excrétion urinaire et l'autre de recyclage. Le contenu de la vessie n'est pas considéré comme un compartiment mais comme le réservoir d'excrétion.

Le tissu osseux est subdivisé en régions corticales (os compact) et trabéculaires (os spongieux) qui ne sont pas à la même distance de la moelle osseuse. La publication 70 (ICRP, 1995) précise l'histologie du squelette. L'activité atteignant le squelette est d'abord déposée sur les surfaces osseuses corticales et trabéculaires puis ensuite transférée soit à la moelle osseuse par résorption de l'os, soit au volume osseux au fur et à mesure de sa formation. L'importance de cette distinction réside dans le fait que les cellules à risque de ce tissu sont situées uniquement dans une couche fine de $10 \mu \mathrm{m}$ d'épaisseur et que parmi 
celles-ci, les cellules endostéales sont situées au voisinage de la moelle osseuse. L'exposition de la moelle hématopoïétique et des surfaces osseuses est largement prédominante dans le calcul de la dose efficace. Chez l'adulte, on utilise les facteurs que l'on observe à l'âge de 25 ans (et non 20 comme pour les autre organes) ; en effet, on considère que les constructions osseuses ne sont stabilisées qu'à partir de cet âge. L'intégration de la dose est donc ici calculée sur la durée de vie qui suit cet âge. Chez l'enfant, les paramètres de fixation et de recyclage des différents modèles varient beaucoup en fonction de l'âge, avec des valeurs maximales dues à la croissance entre 0 et 2 ans et entre 12 et 15 ans.

Deux variantes biocinétiques sont modélisées en marquant la différence entre les comportements des actinides et ceux des alcalino-terreux :

Le modèle des actinides (Legget, 1992) pour le plutonium, l'américium, le neptunium et le curium, éléments qui se lient préférentiellement aux surfaces osseuses est décrit dans les publications 67 et 69 (ICRP, 1993 et 1995).

Les biocinétiques de chacun des actinides, fractions respectives retenues et périodes biologiques de transfert entre les divers compartiments, varient pour chaque élément et selon l'âge des sujets. La figure 8 fournit à titre d'exemple les paramètres du plutonium chez l'homme adulte.

Le sang perd son activité avec une période biologique de 0,75 jours : $30 \%$ de l'activité se fixe dans le pool d'échange rapide des tissus mous ST0 d'où elle est libérée rapidement $\left(T_{\mathrm{b}}=1\right.$ jour $), 9 \%$ dans ST1 qu'elle quitte moins vite ( $T_{\mathrm{b}}=4$ ans), et $1,4 \%$ dans ST2, $\left(T_{\mathrm{b}}=100 \mathrm{ans}\right)$. Le foie comporte un (américium) ou deux compartiments (plutonium et neptunium).

Les gonades fixent moins de $1 \%$ de l'activité sanguine, mais cette activité est retenue à long terme $\left(T_{\mathrm{b}}=10 \mathrm{ans}\right)$.

Dans le squelette, l'activité est d'abord fixée massivement par les surfaces osseuses ( $21 \%$ pour $\mathrm{Am}, 32 \%$ pour $\mathrm{Np}$ et $35 \%$ pour $\mathrm{Pu}$ ) ; ses transferts découlent ensuite du processus de construction osseuse : l'activité passe, soit dans les cavités où se trouve la moelle osseuse par résorption des surfaces trabéculaires $\left(T_{\mathrm{b}}=3,8 \mathrm{ans}\right)$ et corticales $\left(T_{\mathrm{b}}=23 \mathrm{ans}\right)$, soit dans le volume de l'os $\left(T_{\mathrm{b}}=7,7\right.$ et 46 ans) et rejoint la moelle à son tour comme précédemment. L'activité de la moelle passe alors au sang, $\left(T_{\mathrm{b}}=90\right.$ jours $)$ pour être redistribuée selon le schéma initial. Au total, par comparaison avec le modèle biocinétique décrit dans la publication 48 (ICRP, 1986) on observe que ce modèle prévoit une rétention à long terme supérieure pour le foie et le squelette (Leggett, 1992).

Le rein, en accord avec la plupart des observations humaines, excrete directement $2 \%$ de l'activité sanguine immédiatement et $1 \%$ avec une période de 50 jours. On a ajouté, pour le plutonium, une excrétion directe de la moitié de la fraction ST1 ( $T_{\mathrm{b}}=1450$ jours). Dans le second compartiment rénal, $0,5 \%$ de l'activité sanguine est retenue puis ensuite transférée au sang ( $T_{\mathbf{b}}=500$ jours $)$. 


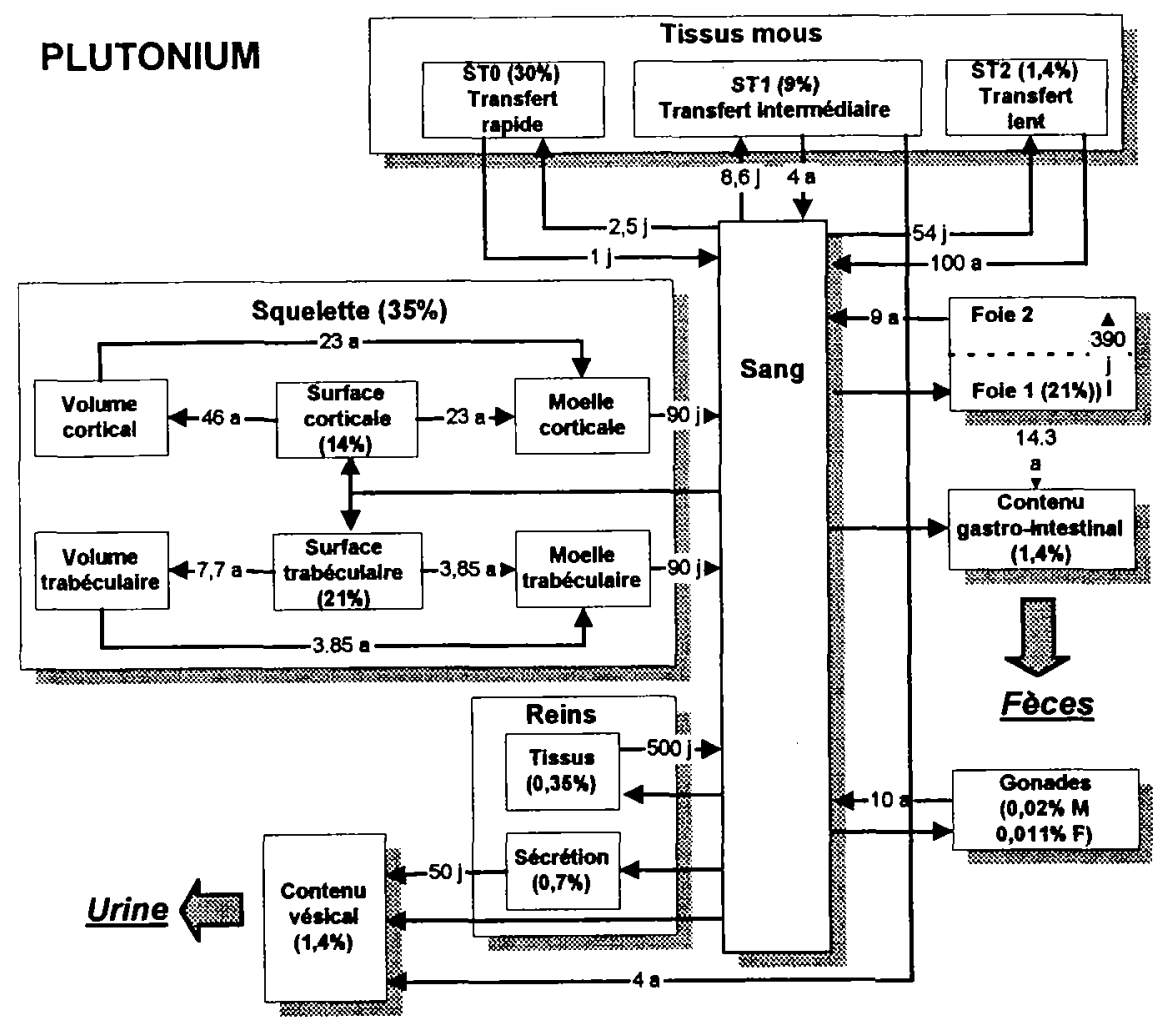

Organe $(X \%)=$ fraction transférée du sang à l'organe en pourcentage du total

Fig. 8. - Modèle biocinétique du plutonium CIPR 67 (adulte).

Actinides model in adults : plutonium, from Publication 67(ICRP 1993).

L'excrétion urinaire prévue par ce modèle est prolongée à plus long terme que ne le prévoyait les fonctions d'excrétion de la publication 54 (ICRP, 1988).

Le modèle des alcalino-terreux, strontium, baryum et radium, éléments qui se lient plutôt au volume de l'os (Legget, 1994).

Ce terme générique ne doit pas être pris trop littéralement : en effet, il est aussi décrit pour le plomb dans la publication 67 (ICRP, 1993) et l'uranium et le thorium dans la publication 69 (ICRP, 1995). La figure 9 donne à titre d'exemple les paramètres de l'uranium. 


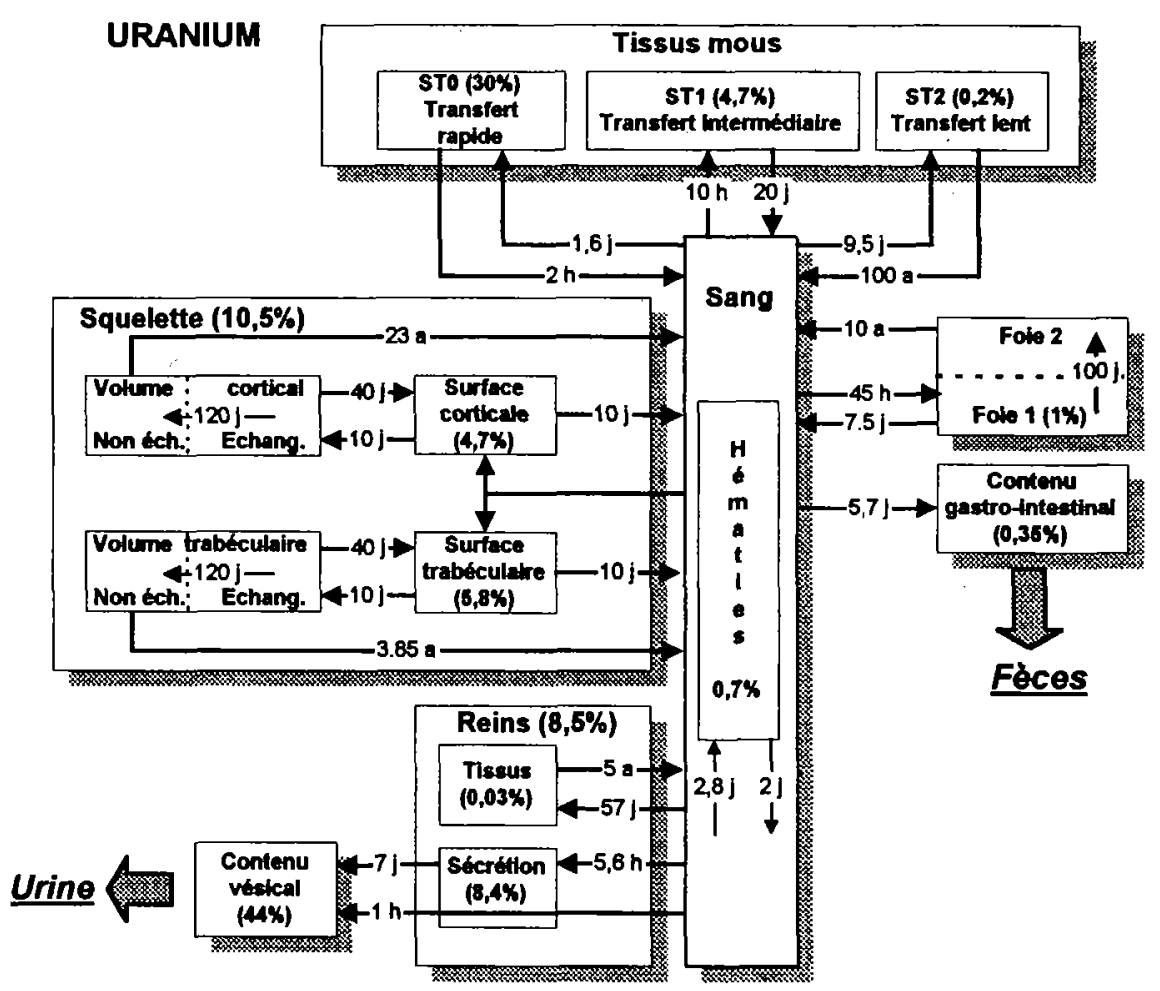

Organe $(X \%)=$ fraction transférée du sang à l'organe en pourcentage du total

Fig. 9. - Modele biocinétique des alcalino-terreux: exemple de l'uranium chez l'homme adulte (CIPR 69).

Rare earth model in adults : uranium, from publication 69 (ICRP, 1995).

Les principales différences avec celui des actinides sont les suivantes :

Dans les tissus mous, la fraction à échange rapide avec le sang $\left(\mathrm{ST}_{0}\right)$ l'emporte largement. Le reste de l'activité plasmatique est directement éliminé dans les excrétions : essentiellement dans les urines pour le $\mathrm{Sr}$, dans les selles pour le Ba et le Ra.

La fixation dans le foie est nulle $(\mathrm{Sr}, \mathrm{Ba})$ ou très faible $(1 \%$ pour l'uranium et pour le radium) mais la fixation initiale dans les autres tissus mous est importante $(60 \%$ pour le strontium, $30 \%$ pour l'uranium et 42 à $43 \%$ pour le baryum et le radium). 
Comme l'épuration des tissus mous est plus rapide que celle de l'os, quelques mois après, toute l'activité résiduelle contenue dans l'organisme se retrouve dans l'os par croissance et remodelage de celui-ci.

La fixation initiale dans le squelette représente 25 à $30 \%$ de l'activité présente dans le plasma. L'activité sanguine qui atteint le squelette, est déposée de façon transitoire sur les surfaces osseuses pour être redistribuée au sang ou transférée au volume de l'os; dans ce volume, elle se répartit entre deux compartiments, os trabéculaire et cortical, eux-mêmes divisés chacun en deux : un premier échangeable et un deuxième non échangeable. L'activité du premier va en partie vers les surfaces et en partie vers le deuxième. Celle du second retourne finalement au sang par résorption osseuse sans passage par le compartiment médullaire, contrairement aux actinides.

Au total, si l'on compare les courbes de rétention dans les organes entiers obtenues avec ce modèle et avec celui de la CIPR 30 (ICRP, 1979) on observe qu'elles sont similaires pour le rein et le squelette tandis que les autres tissus, en particulier le foie, montrent une rétention plus longue (Leggett, 1994).

\subsubsection{Le plomb}

Le modèle du plomb, dérivé de celui des alcalino-terreux, est décrit dans la publication 67 (ICRP, 1993). Le plomb se fixe au niveau du volume osseux mais la fraction de l'activité plasmatique, initialement fixée, est deux fois plus faible $(12,5 \%)$. La fixation initiale dans le foie n'est plus négligeable $(7 \%)$ et celle dans les autres tissus mous du même ordre de grandeur que pour le Ba et le Ra. L'excrétion initiale totale est seulement de $3 \%$ de l'activité plasmatique. L'épuration de l'os et des tissus mous est plus. lente que pour les alcalino-terreux.

La particularité du plomb est l'individualisation, comme pour le fer dans le compartiment sanguin, d'un sous compartiment correspondant aux globules rouges. Ils fixent $40 \%$ de l'activité qui pénètre dans le plasma. Cette activité retourne dans le plasma avec une période de cinq jours.

Le plomb présente la spécificité d'avoir d'autres modalités d'excrétion que les urines et les selles : $0,6 \%$ de l'activité plasmatique est éliminée dans la sueur et $40 \%$ de l'activité fixée dans le compartiment d'épuration intermédiaire des tissus mous (ST1) sont éliminés essentiellement dans les cheveux mais aussi les ongles et les squames épidermiques.

\subsubsection{Comportement biocinétique des produits de filiation}

Dans les modèles décrits, l'approche générale de la publication 30 (ICRP, 1979) fait l'hypothèse que les descendants ont le même comportement biocinétique que leurs parents, mais il existe plusieurs cas particuliers, par exemple :

- les descendants iodés du tellure suivent le modèle biocinétique thyroïdien de l'iode. 
- le radon descendant du radium (par exemple le ${ }^{222} \mathrm{Rn}$, descendant du radium 226) suit un modèle particulier complexe :

- sous forme de gaz, il est exhalé à partir des tissus mous et du sang avec un taux constant de $1 \mathrm{~min}^{-1}$ et retenu à $30 \%$ dans l'os,

- sous forme de descendants solides, à partir des tissus mous et des surfaces osseuses, il est transféré au sang à un taux de $100 \mathrm{j}^{-1}$,

- à partir des surfaces osseuses, il migre des compartiments échangeables et non échangeables avec des taux respectifs de $1,5 \mathrm{j}^{-1}$ et de $0,36 \mathrm{j}^{-1}$.

En ce qui concerne les éléments à vie courte (quelques minutes) il est admis qu'ils disparaissent sur leur site d'origine.

\subsection{Calcul de dose après une incorporation}

Les doses dues à une incorporation d'un radionucléide sont strictement proportionnelles à l'activité incorporée. Les facteurs de proportionnalité sont respectivement $H_{50}$,T pour la dose équivalente engagée à l'organe $\mathrm{T}$ et DPUI pour la dose efficace.

La détermination de l'activité incorporée s'effectue à partir des mesures de rétention et d'excrétion du sujet en comparant les activités mesurées de chaque radionucléide détecté avec les activités théoriques, calculées à l'aide des modèles (rétentions et excrétions théoriques par unité d'activité incorporée). La publication 54 (ICRP, 1988) contient sous forme de tableaux et de courbes la valeur des rétentions et des excrétions théoriques en fonction du temps après une incorporation par inhalation de $1 \mathrm{~Bq}$ de différents radionucléides. Chaque mesure effectuée peut donc fournir, pour chaque radionucléide, une estimation de l'activité inhalée en divisant l'activité mesurée par l'activité théorique correspondante (même type de mesure, même temps écoulé depuis l'inhalation).

La cohérence des différentes estimations obtenues permet de tester la validité du modèle utilisé (date d'inhalation, classe de transférabilité, DAMA). Plus l'activité inhalée est importante, plus il paraît souhaitable de multiplier dans le temps le nombre et le type de mesures effectuées afin d'être à même de pouvoir confirmer ou modifier le modèle initialement adopté. La meilleure estimation possible de l'activité inhalée sera la moyenne des estimations obtenues à partir des différentes mesures.

La dose équivalente engagée à l'organe le plus exposé ou la dose efficace pourront ensuite être calculées en multipliant l'activité moyenne inhalée, respectivement par les coefficients de dose $H_{50}$, et DPUI, correspondant aux paramètres du modèle adopté pour l'estimation de l'activité inhalée.

Les paramètres des modèles utilisés interviennent à la fois dans le calcul des coefficients de dose et dans celui des rétentions et des excrétions fractionnelles théoriques. Pour celles-ci, l'utilisation du nouveau modèle pulmonaire rendra donc obsolète l'ensemble des données de la publication 54 (ICRP, 1988) en 
particulier celles qui permettent d'interpréter en termes d'activité inhalée le résultat des mesures de rétention et d'excrétion effectuées. Ce nouveau modèle sera applicable pour le calcul des rétentions et des excrétions lorsque sera parue la version révisée $54 \mathrm{R}$ de la publication 54.

\subsection{Limites Annuelles d'Incorporation (LAI)}

Pour les radionucléides pour lesquels on connaît les coefficients de dose par unité d'activité incorporée $\left(H_{50}, \mathrm{~T}, \mathrm{DPUI}\right)$, il est possible de calculer les activités incorporées qui correspondent aux limites primaires réglementaires, exprimées en termes de dose. Ces activités incorporées sont appelées Limites Annuelles d'Incorporation (LAI). Actuellement, les limites annuelles primaires, pour les travailleurs, sont de $50 \mathrm{mSv}$ à l'organisme entier pour limiter le risque de survenue d'un effet stochastique et de $500 \mathrm{mSv}$ à l'organe le plus exposé pour empêcher la survenue d'un effet de type déterministe. La LAI est la valeur la plus basse qui satisfasse les deux relations :

$$
\operatorname{LAI}(\mathrm{Bq})=\frac{0,05(\mathrm{~Sv})}{\mathrm{DPUI}(\mathrm{Sv} / \mathrm{Bq})} \quad \text { et } \quad \mathrm{LAI}=\frac{0,5}{H_{50, \mathrm{~T}}}
$$

Comme les DPUI et $H_{50}$,T , les LAI sont calculées suivant différentes hypothèses: incorporation par ingestion (différentes valeurs de $f_{1}$ ) ou inhalation (différentes classes de transférabilité, DAMA $=1 \mu \mathrm{m}$ ). Ce sont des limites secondaires. Elles figurent encore dans la réglementation actuelle.

Les LAI constituent des repères commodes car elles permettent, en pratique, de comparer la radiotoxicité relative de différents radionucléides. Mais le calcul des doses après une incorporation se fait directement à partir des DPUI (Vennart, 1981).

Les LAI présentent, par contre, l'inconvénient majeur d'être exprimées en becquerels. Elles sont souvent comparées aux rétentions mesurées par l'anthropogammamètre exprimées aussi en becquerels. Le rapport $I / \mathrm{LAI}$, préconisé par la réglementation, où $I$ est l'activité incorporée calculée, est alors interprété de manière erronée par : rétention/LAI. Il existe un autre inconvénient à exprimer la réglementation en termes de limites secondaires et non de limites primaires, c'est de manquer de souplesse pour l'interprétation des mesures effectuées sur les individus.

Dans la Directive Européenne du 13 mai 1996, les limites sont seulement des limites primaires. Pour l'exposition interne, les LAI ont disparu et on a seulement indiqué les doses efficaces par unité d'incorporation, DPUI.

\subsection{Limites dérivées de concentration dans l'air (LDCA)}

Comme son nom l'indique, la LDCA est une limite dérivée opérationnelle, utilisée pour la surveillance des lieux de travail. Les concentrations mesurées dans l'air des locaux peuvent lui être directement comparées. 
La LDCA est, pour un radionucléide, la concentration moyenne inhalée, exprimée en $\mathrm{Bq} \mathrm{m}{ }^{-3}$, qui pour 2000 heures de travail par an, entraîne une incorporation égale à la LAI par inhalation.

$$
\operatorname{LDCA}\left(\mathrm{Bq} \mathrm{m}^{-3}\right)=\frac{\mathrm{LAI}(\mathrm{Bq})}{2000(\mathrm{~h}) \times 1,2\left(\mathrm{~m}^{3} \mathrm{~h}^{-1}\right)},
$$

où $1,2 \mathrm{~m}^{3} \mathrm{~h}^{-1}$ est le débit ventilatoire d'un homme effectuant un travail léger.

Les LAI sont des intermédiaires utiles mais non indispensables à la définition et au calcul des LDCA. Celles-ci peuvent être directement calculées à partir des DPUI. Comme les LAI, plusieurs LDCA peuvent correspondre à un même radionucléide suivant la solubilité des composés manipulés dans les différents locaux. Un autre paramètre qui intervient implicitement dans le calcul des LDCA est le DAMA des aérosols inhalés. Ce DAMA est variable : dans les ateliers, la plupart des mesures effectuées ont montré qu'il était généralement supérieur au diamètre de $1 \mu \mathrm{m}$ admis actuellement ; à l'inverse, pour les opérations de démantèlement, sont générés des aérosols très fins dont les particules atteignent des tailles pouvant aller jusqu'à $10^{-3} \mu \mathrm{m}$.

Au total, pour la surveillance des ambiances, il paraît raisonnable que sur les lieux de travail soient effectuées, par des spécialistes, des mesures des diamètres aérodynamiques et de la solubilité des particules en suspension dans l'air. De telles mesures devraient être contrôlées périodiquement, en particulier lorsque interviennent des modifications des procédés de fabrication. Ces mesures permettraient de calculer des $L D C A$ spécifiques plus réalistes. Dans la réglementation pourraient figurer des LDCA ou $\mathrm{DCA}^{(1)}$ qui seraient prises par défaut lorsque de telles mesures ne sont pas effectuées.

Les mesures d'ambiance doivent compléter mais non se substituer au suivi individuel de l'exposition interne, en particulier pour les travailleurs de catégorie A.

\section{7. Évolutions récentes des doses par unité d'incorporation recommandées par la CIPR}

C'est en utilisant les récentes valeurs des $f_{1}$, les modèles métaboliques systémiques révisés et le nouveau modèle pulmonaire de la publication 66 (ICRP, 1994) que l'on vient de remplacer par la publication 68 (ICRP, 1994) la publication 61 (ICRP, 1991) donnant des valeurs de LAI, maintenant caduques. Ce document fournit pour le travailleur les nouvelles DPUI pour les différents radionucléides.

(1) Dans les publications de la CIPR le terme « limite " n'est pas associé à la concentration dérivée dans l'air. 
Pour l'inhalation, les DPUI, qui figurent dans la publication 68 (ICRP, 1994) sont calculées pour les aérosols rencontrés dans l'environnement des lieux de travail avec la valeur de $5 \mu \mathrm{m}$ comme DAMA. Les DPUI pour $1 \mu \mathrm{m}$, taille précédemment utilisée, sont aussi fournies pour faciliter la comparaison avec les LAI de la publication 61 (ICRP, 1994). Les DPUI ainsi calculées sont souvent plus basses que celles qui étaient obtenues avec le modèle pulmonaire de la publication 30 (ICRP, 1979) ; ceci est dû surtout à des valeurs de dépôt revues à la baisse, principalement dans la région alvéolo-interstitielle.

Pour les gaz inhalés, les DPUI ont été revues pour les radionucléides déjà présents dans la publication 61 (ICRP, 1994) ainsi que pour quelques autres (tels que l'eau tritiée et le tritium organique gazeux, le carbonyle nickel, l'iode, le mercure, l'argon, le krypton, le xénon, etc. ) en fonction de leur solubilité dans les voies aériennes et de leur réactivité avec ces tissus.

\section{Conclusion}

Les DPUI de la publication 68 (ICRP, 1994) sont spécifiques des travailleurs. Les valeurs correspondantes pour les personnes du public, adultes et enfants de 3 mois, 1, 5, 10 et 15 ans sont disponibles pour l'ingestion dans les publications 67 et 69 (ICRP, 1993 et 1995) et pour l'inhalation dans la publication 71 (ICRP, 1995). Les doses efficaces par unité d'incorporation données dans ces trois dernières publications ont été compilées pour la publication 72 (CIPR, 1996). Plusieurs versions sur cd-rom en seront bientôt également disponibles. Toutes ces valeurs sont reprises dans les principaux standards internationaux : en particulier par l'Agence internationale de l'énergie atomique pour la publication des «Basic Safety Standards » (AIEA, 1996). Elles ont servi également de base à l'élaboration de la Directive européenne du 13 mai 1996 sur les normes de radioprotection. La révision de la publication 54 (ICRP, 1988) donnera bientôt sous le $\mathrm{n}^{\circ} 54 \mathrm{R}$, les valeurs des fonctions de rétention dans le corps entier et d'excrétions urinaire et fécale, découlant des modèles biocinétiques récents. Dans les années qui viennent, les nouveaux modèles actualiseront ceux de la publication 30 (ICRP, 1979) qui sera alors remplacée.

\section{Annexe. Mode de calcul des dose engagées aux organes et à l'orga- nisme entier.}

\section{A.1. Dose équivalente engagée à un tissu ou un organe}

Lorsqu'une substance radioactive a été fixée dans un organe ou un tissu, cet organe devient une source d'exposition pour lui-même et les tissus voisins $(T)$.

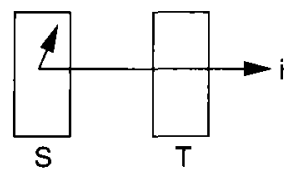


La dose équivalente absorbée dans $\mathrm{T}$, due à l'émission d'un rayonnement $i$, lors d'une désintégration dans l'organe source (S), est égale au produit de l'énergie cédée par kilogramme de tissu $\mathrm{T}$ (dose absorbée moyenne) et du facteur de pondération du rayonnement $\left(W_{\mathrm{R}}\right) i$. Cette grandeur est notée SEE $(\mathrm{T} \leftarrow \mathrm{S}) \quad i$ dans les publications de la CIPR. Elle est exprimée en $\mathrm{MeV} \mathrm{g}^{-1}$ désintégration ${ }^{-1}$. Son expression en sievert-désintégration-1 nécessite de la multiplier par $1,6 \times 10^{-10}\left(1 \mathrm{MeV} \mathrm{g}^{-1}=1,6 \times 10^{-10} \mathrm{j} \mathrm{kg}^{-1}\right)$.

La dose équivalente totale absorbée dans $\mathrm{T}$, due à l'émission du rayonnement $i$ pendant un temps $\tau$ après l'incorporation, est appelée dose équivalente engagée $\left(H_{\mathrm{T}}(\tau)\right)$. Elle est égale au produit de la dose équivalente absorbée par désintégration et du nombre total de désintégrations dans l'organe $\mathrm{S}$ pendant le temps $\tau\left(U_{\mathrm{S}}\right)$ :

$$
H_{\mathrm{T}}(\tau)=1,6 \times 10^{-10} \mathrm{SEE}(\mathrm{T} \leftarrow \mathrm{S}) i \times U_{\mathrm{S}}(\tau),
$$

$\tau$ est pris égal à 50 ans pour les adultes et à 70 ans pour les enfants.

La dose équivalente engagée totale dans l'organe $\mathrm{T}$ sera la somme des doses équivalentes engagées dues aux différents rayonnements émis par le ou les radionucléides contenus dans les différents organes sources et dans l'organe $T$ lui-même. Au total, la plus grande part de cette dose sera due aux rayonnements peu pénétrants $(\alpha, \beta)$ émis dans T et dont l'énergie sera absorbée dans T.

Dans la publication 30 de la CIPR (1979) figure pour chaque radionucléide et pour une incorporation par ingestion et par inhalation (ancien modèle pulmonaire, DAMA $=1 \mu \mathrm{m}$, classes $\mathrm{D}, \mathrm{W}, \mathrm{Y}$ ) le résultat du calcul du nombre de désintégrations en 50 ans dans différents organes $\left(U_{\mathrm{S}}\right)$. Ce calcul est effectué par intégration de la fonction de rétention correspondante.

Les différentes valeurs des SEE $(\mathrm{T} \leftarrow \mathrm{S})$ sont également fournies ainsi que les valeurs des doses équivalentes engagées, par unité d'activité incorporée, dans les différents organes $\left(H_{50}, \mathrm{~T}\right.$ en $\left.\mathrm{Sv} \mathrm{Bq}{ }^{-1}\right)$. Il existe autant de séries de valeurs de $h_{50}$, T que d'hypothèses prises pour leur calcul : incorporation par ingestion (différentes valeurs de $f_{1}$ ), incorporation par inhalation (différentes classes de transférabilité). Seuls figurent dans les tableaux les organes pour lesquels $H_{50}$,T est supérieur à $10 \%$ de la valeur de l'organe le plus exposé.

De plus, dans le cas d'une incorporation par inhalation d'aérosols dont le DAMA est différent de $1 \mu \mathrm{m}$, la publication 30 et la publication 66 de la CIPR fournissent également les indications nécessaires à la correction des $H_{50}$, T correspondants.

\section{A.2. Dose à l'organisme entier. Dose efficace}

Pour une exposition non uniforme de l'organisme, qui est généralement le cas après une incorporation de radionucléides, est défini l'équivalent de dose efficace engagée ou plus simplement la dose efficace. La dose efficace est numériquement égale à la dose équivalente absorbée qui, délivrée lors d'une exposi- 
tion uniforme et brève de l'organisme entier, entraînerait le même risque de survenue d'effets stochastiques. Elle est égale à la somme des doses équivalentes engagées pondérées dans les organes et tissus de l'organisme :

$$
E=\sum_{\mathrm{T}} W_{\mathrm{T}} \cdot H_{\mathrm{T}} .
$$

Le facteur de pondération $W_{\mathrm{T}}$ du tissu ou de l'organe $\mathrm{T}$ représente la contribution relative de $\mathrm{T}$ au détriment total dû aux effets qui résulteraient d'une irradiation uniforme de tout le corps. En d'autres termes, $W_{\mathrm{T}}$ est égal, pour une irradiation uniforme de $1 \mathrm{~Sv}$, à la probabilité de survenue d'un effet stochastique dans l'organe $T$, divisée par la probabilité de survenue d'un effet stochastique dans la totalité de l'organisme. La somme des facteurs de pondération est donc égale à l'unité.

\section{RÉFÉRENCES}

AIEA (1996) International Basic Safety Standards for Protection against Ionizing Radiation and for the Safety of Radiation Sources (Safety series $n^{\circ} 115-I$ ). AIEA, Vienne.

DIRECTIVE 96/29 EURATOM (1996) du Conseil du 13 Mai 1996, fixant les normes de base relatives à la protection sanitaire de la population et des travailleurs contre les dangers résultant des rayonnements ionisants. J. O. C. E. $n^{\circ} 159$ du 29 Juin 1996.

ICRP (1979) Limits for Intakes of Radionuclides by Workers, ICRP publication 30. Pergamon press, Oxford.

ICRP (1986) The metabolism of plutonium and related elements, ICRP publication 48. Pergamon press, Oxford.

ICRP (1987) Radiation Dose to Patients from Radiopharmaceuticals, ICRP publication 53. Pergamon press, Oxford.

ICRP (1988) Individual Monitoring for Intakes of Radionuclides by Workers : Design and Interpretation, ICRP publication 54. Pergamon press, Oxford.

ICRP (1989) Age Dependent Doses to Members of the Public from Intake of Radionuclides, ICRP publication 56, Part 1. Pergamon press, Oxford.

ICRP (1991) Recommendations of the International Commission on Radiological Protection, ICRP publication 60. Pergamon Press, Oxford.

ICRP (1991) Annual Limits on Intake of Radionuclides by Workers Based on the 1990 Recommendations, ICRP publication 61. Pergamon Press, Oxford.

ICRP (1994) Human Respiratory Tract Model for Radiological Protection, ICRP publication 66. Elsevier Science Ltd, Oxford.

ICRP (1993) Age Dependent Doses to Members of the Public from Intake of Radionuclides: Part 2. Ingestion dose coefficients, ICRP publication 67. Elsevier Science Ltd, Oxford.

ICRP (1994) Dose Coefficients for Intakes of Radionuclides by Workers (ICRP publication 68). Elsevier Science Ltd, Oxford.

ICRP (1995) Age Dependent Doses to Members of the Public from Intake of Radionuclides : Part 4. Ingestion dose coefficients, ICRP publication 69. Elsevier Science Ltd, Oxford. 
ICRP (1995) Basic anatomical and physiological data for use in Radiological Protection : the skeleton, ICRP publication 70. Elsevier Science Ltd, Oxford.

ICRP (1995) Age Dependent Doses to Members of the Public from Intake of Radionuclides : Part 4. Inhalation dose coefficients, ICRP publication 71. Elsevier Science Ltd, Oxford.

ICRP (1996) Age Dependent Doses to Members of the Public from Intake of Radionuclides : Part 5. Compilation of ingestion and inhalation dose coefficients, ICRP publication 72. Elsevier Science Ltd, Oxford.

Jarvis N.S., Birchall A. (1994) LUDEP 1.0, un logiciel pour ordinateur personnel qui applique le nouveau modèle de la CIPR. Radioprotection, 29, 81-86.

Leggett R.W. (1992) A retention-excretion model for americium in humans. Health Phys., 62, 288-310.

Leggett R.W. (1994) Basis for the ICRP's age-specific biokinetic model for uranium. Health Phys., 67, 589-610.

Piechowski J., Menoux B., Chaptinel Y. (1992) Evaluation de l'exposition systémique résultant d'une blessure contaminée par des produits radioactifs. Rapport CEA - R5583.

Robertson R.R., Price R.R., Budinger T.F., Fairbanks V.F., Pollycove M. (1983) Radiation absorbed doses from Iron 52 and Iron 59 used to study ferrokinetics. MIRD Dose Est. Rep. 11, J. Nucl., 24, 339-348.

Simko M., Noßke D. (1996) Basis for a revision of the gastrointestinal tract model. Health Phys., 63, 29-36.

Stubbs J.B. (1992) Results from a new mathemetical model of gastrointestinal transit that incorporates age and gender dependent physiological parameters. Rad. Prot. Dosim., 41, 63-69.

Vennart J. (1981) Limits for intakes of radionuclides by workers, ICRP publication 30. Health Phys., 40, 477-484. 